\title{
The phenomenological approach to modeling the dark energy
}

\author{
Martin Kunz \\ Département de Physique Théorique and Center for Astroparticle Physics, Université de \\ Genève, Quai E. Ansermet 24, CH-1211 Genève 4, Switzerland \\ E-mail: martin.kunz@unige.ch
}

\begin{abstract}
In this mini-review we discuss first why we should investigate cosmological models beyond $\Lambda$ CDM. We then show how to describe dark energy or modified gravity models in a fluid language with the help of one background and two perturbation quantities. We review a range of dark energy models and study how they fit into the phenomenological framework, including generalizations like phantom crossing, sound speeds different from $c$ and non-zero anisotropic stress, and how these effective quantities are linked to the underlying physical models. We also discuss the limits of what can be measured with cosmological data, and some challenges for the framework.
\end{abstract}

Keywords: cosmology, dark energy, general relativity, cosmological constant, cosmological perturbation theory 


\section{Contents}

1 Introduction $\quad 1$

2 Initial questions $\quad 2$

2.1 Reliability of the data 2

2.2 Why go beyond $\Lambda$ ? 3

2.3 Other possible explanations 5

3 A phenomenological description $\quad 6$

3.1 Overview 6

$\begin{array}{lll}3.2 & \text { Background evolution } & 7\end{array}$

$\begin{array}{lll}3.3 & \text { Perturbation equations } & 10\end{array}$

$\begin{array}{lll}3.4 & \text { Different descriptions } & 12\end{array}$

3.5 Schematic Measurements 14

4 Dark energy models $\quad 14$

4.1 Quintessence 14

4.2 Generalizing Quintessence 15

$\begin{array}{lll}4.3 & \text { Phantom crossing } & 17\end{array}$

4.4 Perturbation evolution in generalized Quintessence models 19

4.5 Observational constraints on the Quintessence equation of state 23

4.6 Modified Gravity models and anisotropic stresses 26

5 Limitations and challenges $\quad \mathbf{3 0}$

$\begin{array}{lll}5.1 & \text { The dark degeneracy } & 30\end{array}$

5.2 Non-linear evolution 33

5.3 Dependence on the environment 34

6 Conclusions and Outlook $\quad 34$

\section{Introduction}

2011 has been an exciting year for dark energy: In the same week in early October, the Nobel prize in physics was given to Saul Perlmutter, Brian P. Schmidt and Adam G. Riess "for the discovery of the accelerating expansion of the Universe through observations of distant supernovae" and the EUCLID satellite mission ${ }^{1}$ [1] was selected by ESA for the second Cosmic Vision launch slot (expected for 2019 - but some delays are likely between now and then). Dark energy has arrived in the main stream of physics, not only cosmology, and it is seen as one of big challenges facing the discipline at the beginning of the 21st century. And at least observationally it really is a 21 st century problem. While theoretically the cosmological constant has been around since the development of General Relativity $[2,3]$ - as the unique additional term that can self-consistently be added to the Einstein equations, and because it would have permitted the construction of a static universe - the two observational publications for which the Nobel prize was given date from 1998 and 1999 respectively [4, 5].

\footnotetext{
${ }^{1}$ Continuously updated information on EUCLID is available on http://www.euclid-ec.org.
} 
Even though there were observational hints before then, it was really the evidence presented in these two papers that led, in a way reminiscent of a phase transition, to the general acceptance amongst cosmologists that some kind of Dark Energy was necessary.

There are several excellent reviews discussing dark energy in great detail, for example [6-12] just to name a few, and there is also a new book by Luca Amendola and Shinji Tsujikawa on the topic [13]. In this mini-review, loosely based on an overview talk given at PONT 2011 in Avignon, I do not plan to compete with these weighty tomes and to discuss in depth the dark energy in all its guises and with all its consequences. Instead, I will present a rather personal view of the dark energy, focused on its phenomenological aspects, and I will be trying to add a few ideas that in my opinion are interesting but a bit off the trodden path. Many more aspects of the accelerating universe are discussed in the companion papers to this one: Observational Evidence of the Accelerated Expansion of the Universe by Pierre Astier and Reynald Pain [14], Everything You always Wanted to Know about the Cosmological Constant (but Were Afraid to Ask) by Jérôme Martin [15], Establishing Homogeneity of the Universe in the Shadow of Dark Energy by Chris Clarkson [16] and Galileons in the Sky by Claudia de Rham [17].

I will start in section 2 with the question whether the data is really reliable and needs to be taken seriously, why it makes sense to go beyond a cosmological constant, and what other explanations beyond $\Lambda$ we can think of.

In section 3 we will then introduce the phenomenological framework that describes the most general fluid in terms of the variables present in the energy-momentum tensor. These variables allow for general departures from $\Lambda \mathrm{CDM}$ and in addition they allow to understand in a precise, quantitative sense, which degrees of freedom can be probed by cosmological measurements.

We review actual dark energy models in section 4, illustrating how they fit into the framework and how different types can be distinguished through the values taken by their fluid parameters. We also discuss how the phenomenological variables allow for straightforward generalizations of the models (for example to different sound speeds) and how this is then reflected in "model space".

In the penultimate section 5 we discuss some of the challenges and limits of the phenomenological description, including the dark degeneracy which illustrates a fundamental limitation of the framework that is a reflection of fundamental limitations of cosmological measurements when trying to reconstruct the physics of the different constituents of the Universe. We conclude this mini-review with a summary and an outlook on future developments in section 6 .

\section{Initial questions}

\subsection{Reliability of the data}

The first question one encounters when thinking about the dark energy is whether the observational evidence is really compelling, or whether the best explanation is that the data is simply wrong. The current observational situation being discussed in detail elsewhere in this volume [14], I will here just highlight a single point, the use of distance duality to check the internal consistency of the distance data. The distance data is important since it provides currently the most precise measurements that only rely on the behavior of the average, homogeneous metric. It is also of historic interest since it is this type of data that garnered the 2011 Nobel prize in physics. 
In any metric theory of gravity, the distances are essentially unique, thanks to a theorem proved in 1933 by Etherington that shows that for this class of theories the relation between luminosity distance and angular diameter distance,

$$
\frac{d_{L}(z)}{d_{A}(z)}=(1+z)^{2}
$$

(where $z$ is the redshift) always holds [18]. This means that we can test the luminosity distance data with the help of angular diameter distance data, and vice versa [19-21]. Since the two types of data agree well even for today's precise measurements [22] this limits a wide range of systematic effects that affect only one of the two distances. Examples on the luminosity distance side include explanations for the observed dimming of supernovae like replenishing grey dust or ultralight axions [23]. More generally speaking, it is very difficult to explain the supernova data by doing unpleasant things to the photons, since this would generically break the distance duality. This leaves effectively only the option to change the behavior of the metric, which is of course exactly what dark energy models do (but see also [24] for interesting conclusions on the metric with the help of distance duality). For this reason it is very unlikely that the distance data is "just" wrong.

\subsection{Why go beyond $\Lambda$ ?}

The cosmological constant dates back right to the development of General Relativity and has been in and out of favor several times during the last century. But, as we will see later on, the data is fully compatible with the dark energy being a cosmological constant, so why should we bother to look elsewhere? There is a whole review in this volume on the cosmological constant [15], so I will only mention here briefly the two main objections, the naturalness problem and the coincidence problem. The first one appears because all particles should give rise to quantum mechanical zero-point fluctuations $\sim \sqrt{k^{2}+m^{2}}$ (with the fermions having the opposite sign to the bosons). Since observations indicate that the energy scale of the cosmological constant would have to be of the order of $10^{-3} \mathrm{eV}$, we can integrate out the heavier particles like electrons. The integral of the zero-point fluctuations over momentum $k$ results in huge contributions to the total cosmological constant (that can in principle be absorbed into a renormalization of a bare cosmological constant) as well as a logarithmic term (see e.g. $[25,26]$ for a recent discussion). Especially the logarithmic contribution is highly problematic: Such terms should be considered physical as they have been observed for masses and couplings, but their contribution to the vacuum energy, already from supposedly well-known particles like the electron, are far larger than the observed value of the cosmological constant. The one known mechanism to suppress the contribution from the zero-point fluctuations, supersymmetry (which relates fermions and bosons to each other), is necessarily broken below a $\mathrm{TeV}$ or else we would already have observed its effects with the help of particle accelerators.

The second issue, the coincidence problem, comes from the fact that if we look at the relative energy density in the cosmological constant during the evolution of the Universe, $\Omega_{\Lambda}(a)=\rho_{\Lambda} / \rho_{\text {tot }}$, we find that there is a sharp transition around today where $\Omega_{\Lambda}(a)$ increases from zero to one. It appears very unlikely that we are living just during this rapid transition, rather than earlier or later. Let us illustrate the coincidence problem a bit more. Writing just $\Omega_{\Lambda}$ for $\Omega_{\Lambda}(a=1)$ we find that close to today this function behaves as

$$
\Omega_{\Lambda}(a) \approx \frac{\Omega_{\Lambda}}{\Omega_{\Lambda}+\left(1-\Omega_{\Lambda}\right) e^{-3 N}},
$$


where $N=\ln (a)$ is the number of e-foldings relative to today, and $\Omega_{\Lambda} \approx 0.73$. Let us call the transition the period from $\Omega_{\Lambda}(a)=0.05$ to $\Omega_{\Lambda}(a)=0.95$. We find that this corresponds to $N \in[-1.31,0.65]$, i.e. in total about two e-foldings, or an expansion of the Universe by a factor of 7.4. If we were more conservative and defined the transition to go from $\Omega_{\Lambda}(a)=0.01$ to $\Omega_{\Lambda}(a)=0.99$ then the transition takes about three e-foldings, corresponding to an expansion by a factor of 20 . I leave it to the reader to judge whether we should consider this very fine-tuned compared to seven e-foldings (factor of 1100) from the emission of the cosmic microwave background radiation $(\mathrm{CMB})$ to today, or the roughly 74 e-foldings between the Planck time and today.

These problems notwithstanding, it is still the case that the cosmological constant is in agreement with all the data, and in addition it remains the best-motivated model. Is it really worth investigating other possibilities? Apart from the basic truth that in order to test a model, we need to go beyond it, there is another reason why I think that the study of dark energy is worthwhile: according to the cosmological standard model, the current period of accelerated expansion is not the first one. The standard model postulates that in the very early Universe, it was also accelerated expansion, called inflation, that created the seeds of the structure that we see around us. Was inflation due to a cosmological constant? It cannot have been a "true" cosmological constant, since inflation ended. But can we test whether it was a kind of pseudo-static vacuum energy, or something dynamical?

Inflationary dynamics is usually parameterized in terms of slow-roll parameters, with the first two (in the Hamilton-Jacobi formalism, see e.g. [27]) defined as

$$
\epsilon_{H}=2 M_{\mathrm{Pl}}^{2}\left(\frac{H^{\prime}}{H}\right)^{2}, \quad \eta_{H}=2 M_{\mathrm{Pl}}^{2} \frac{H^{\prime \prime}}{H} .
$$

Here ' denotes a derivative with respect to the scalar field $\phi$ and we used the reduced Planck mass $M_{\mathrm{Pl}}^{2}=1 /(8 \pi G)$. With $H^{\prime}=\dot{H} / \dot{\phi}$ and with the help of the Friedmann equation (3.4) and the conservation equation (3.5) as well as the expressions for energy density and pressure of a canonical scalar field (4.2) we find $\dot{\phi}=-2 M_{\mathrm{Pl}}^{2} H^{\prime}$ and

$$
1+w=\frac{2}{3} \epsilon_{H} .
$$

The equation of state during inflation is therefore directly given by the first slow-roll parameter. To lowest order in slow-roll this is also related to the tensor to scalar ratio by $r=16 \epsilon_{H}$. Without any further work we can deduce that, since primordial gravitational waves have not been observed (yet?), there is no observational requirement for a deviation from $w=-1$ during inflation. The upper limit on $r$ from the seven-year Wilkinson Microwave Anisotropy Probe (WMAP) data for a flat $\Lambda \mathrm{CDM}$ model without running is about 0.36 [28], corresponding to a maximum deviation from $w=-1$ of 0.015 , and adding additional data would tighten this limit further.

So far it looks like inflation supports also an effective cosmological constant, with $w=$ -1 at the percent-level. However, we can do better by looking at the spectrum of scalar perturbations: the scalar spectral index $n_{s}$ is given to lowest order in slow roll by

$$
n_{s}-1=2 \eta_{H}-4 \epsilon_{H} .
$$

A deviation of the scalar spectral index from the Harrison-Zel'dovich (HZ) case $\left(n_{s}=1\right)$ can be caused by the second slow-roll parameter $\eta_{H}$. Thus even if at a given time $\epsilon_{H} \approx 0$, it is 
still possible to obtain $n_{s} \neq 1$ through a non-zero $\eta_{H}$. But the rate of change of $w$ is also linked to the first two slow-roll parameters,

$$
\frac{d \ln (1+w)}{d N}=\frac{d \ln \epsilon_{H}}{d N}=-2\left(\eta_{H}-\epsilon_{H}\right)
$$

where $N=\ln a$ is again the number of $e$-foldings. Hence if Planck confirms the deviation from HZ that WMAP sees at the $2.5 \sigma$ level, then not both $\epsilon_{H}$ and $\eta_{H}$ can be zero. And if not both are zero, then either $w \neq-1$ or $w$ is changing with time during inflation, or both. In either case, the result is not compatible with an effective cosmological constant for the time period when the observable scales left the horizon, and we will be able to conclude that inflation was driven by a kind of early dynamic dark energy. The Planck measurement of $n_{s}$ may therefore lead to the first direct experimental evidence of the existence of dark energy dynamics [29].

\subsection{Other possible explanations}

If we accept that the data is indeed showing a surprising behavior of the Universe and is not just plain wrong, and that we should look beyond a cosmological constant (at the very least in order to test the $\Lambda$ CDM model), what possible explanations are there? Basically, we have to revisit the (sometimes implicit) assumptions that we have made and judge whether they were reasonable and if there is a way to test them. We should not forget though that all these alternative explanations also need to explain why the cosmological constant is sufficiently small as to be irrelevant, when naively we would expect it to be far too large.

There are at least three fundamental assumptions that we usually make for the $\Lambda$ CDM model:

1. The Universe contains the standard model particles, a dark, cold, matter-like component and a cosmological constant.

2. The large-scale evolution of the Universe is described well by General Relativity (GR).

3. The metric is well described by a Friedmann-Lemaitre-Robertson-Walker (FLRW) metric with small fluctuations.

Let us start with the last assumption. Some theoretical justification is given by the Cosmological (or Copernican) Principle that demands that there are no preferred observers in our Universe, implying that it should be close to homogeneous and isotropic. Observationally, the CMB looks very uniform and supports the Cosmological Principle. If the Universe was exactly homogeneous and isotropic then GR implies that the metric is of the FLRW form. A crucial question therefore is whether the cosmological evolution of a state that is initially close to FLRW can induce large changes from the expected evolution of the averaged metric. This scenario is often called backreaction [30-33], and since it is discussed elsewhere [16] I will not consider it further ${ }^{2}$. The only question I want to raise here is why in such a scenario the apparent evolution of the Universe should be close to $\Lambda \mathrm{CDM}$ for all existing probes. It is not obvious why the backreaction scenario should evolve towards a de-Sitter like state. So

\footnotetext{
${ }^{2}$ I include here models that explicitly violate the Copernican Principle through the use of a fundamentally inhomogeneous metric like LTB [34-39] - in my personal opinion, these models are more likely to represent effective descriptions of the backreaction scenario [40] rather than fundamental models themselves, although large voids could potentially have been formed during inflation [41].
} 
although backreaction models can potentially avoid some of the fine-tuning issues inherent in $\Lambda \mathrm{CDM}$, they seem subject to others that are not of a theoretical but of an observational nature.

The other two assumptions are more closely related to the topic of this review, namely that GR may be modified on large scales, and that there may be more in the Universe than the particles that we know about (plus the dark matter). Here I will concentrate on the first assumption, not least motivated by the discussion in the last section, namely that during inflation we seem to observe a behavior that already requires extra ingredients beyond those found in $\Lambda \mathrm{CDM}$. But as we will see, the formalism that we will put in place in the next section is equally able to deal with modifications of GR, at least in an indirect way.

\section{A phenomenological description}

\subsection{Overview}

Given the range of different explanations, it is useful to put in place a general and unified description of possible observations. In this way, we can analyze the data without the need to assume a specific model, and thus without imposing in advance limitations on what can be tested.

The best way to build such a framework is by reconstructing the metric perturbations. Here we will restrict ourselves to scalar perturbations on a flat background for simplicity, but the approach can be generalized (and in general will need to be generalized once we consider non-linear perturbations as then the scalar-vector-tensor split is no longer conserved). The metric perturbations are good variables since they describe all observations that rely on gravity alone, specifically all cosmological probes ${ }^{3}$. Once the metric is given (as a function of space and time), we know that the observations are now also fixed as the metric describes the motion of both light and of the test particles (excluding astrophysical effects on small scales that we will neglect as we concentrate on observations on large scales $k \lesssim 0.1 / \mathrm{Mpc}-$ see sections 5.2 and 5.3 for short remarks concerning non-linear effects on smaller scales).

The basic outline is then as follows: the line element that we will consider is

$$
d s^{2}=a^{2}\left[-(1+2 \psi) d \eta^{2}+(1-2 \phi) d x^{2}\right] .
$$

Here $\eta$ is conformal time, and we will denote the physical time as $t$, with $d t=a d \eta$. We therefore have one scale factor $a(t)$ and two metric potentials $\phi(x, t)$ and $\psi(x, t)$ that we need to know in order to fix the cosmological evolution of the Universe.

Through the Einstein equations

$$
G_{\mu \nu}=8 \pi G_{N} T_{\mu \nu}
$$

we can link the quantities in the metric to a physical description of the constituents of the Universe. We can do this not only for a "typical" dark energy model where an extra component included in the total energy-momentum tensor on the "right hand side" of the Einstein equations is responsible for the accelerated expansion, but also for models where the Einstein equations themselves are modified. Even in that case we can use the observational fact that we are $3+1$ dimensional beings to always project the equations down to $3+1$ dimensions,

\footnotetext{
${ }^{3}$ I exclude here observations of non-gravitational physics like those of spectral lines in quasars that probe the variation of fundamental constants, even though such observations would provide a powerful way to probe the presence of light fields [42]. See e.g. [43] for a review on varying constants and cosmology.
} 
where we can use the (fundamental or induced) metric to construct the Einstein tensor $G_{\mu \nu}$ and then build an effective dark energy-momentum tensor by subtracting the $T_{\mu \nu}$ of the known components (baryons, radiation and neutrinos):

$$
X_{\mu \nu} \equiv M_{\mathrm{Pl}}^{2} G_{\mu \nu}-T_{\mu \nu}
$$

The new effective total energy momentum tensor on the right hand side is conserved (meaning $\left.\nabla_{\mu} X_{\nu}^{\mu}=0\right)$ just because the Einstein tensor $G$ obeys the Bianchi identity $\nabla_{\mu} G_{\nu}^{\mu}=0$ and because the EMT of the known particles satisfies $\nabla_{\mu} \tilde{T}_{\nu}^{\mu}=0$.

How should we interpret this framework? In general, we need to interpret observations within a theory. Without any theory, we do not know at all how to connect the observed distribution and motion of e.g. galaxies with physics. We expect that the correct theory is similar to GR (and quite possibly is GR). So we just start by assuming this to be the case, and derive the consequences that arise from this assumption. If the resulting energy-momentum tensor looks suspicious, then we will have to consider the possibility that GR may have to be modified. We will discuss in section 4.6 what such a suspicious signature of modified gravity could be.

\subsection{Background evolution}

Let us start with a simple example of the framework that is well known and that has been used for a long time: the so-called background evolution of the Universe, i.e. the evolution of the averaged perfectly homogeneous and isotropic Universe. In addition, we take the Universe to be spatially flat for simplicity. In that case, the 0-0 Einstein equation is the Friedmann equation,

$$
\left(\frac{\dot{a}}{a}\right)^{2}=\frac{8 \pi G_{N}}{3} \rho
$$

(with an overdot denoting a derivative with respect to physical time $t$ ), and the conservation equation becomes

$$
\dot{\rho}+3\left(\frac{\dot{a}}{a}\right)(\rho+p)=0 .
$$

In this case we have one free function that describes the geometry, $a$ or $H \equiv \dot{a} / a$, and two functions that describe the contents of the Universe, $\rho$ and $p$. The Einstein equation provides a link between the geometry and the contents. If we knew $\rho$ then we could use it to compute the evolution of $a$. But in order to determine $\rho$ we need to know the pressure $p$. In this scheme it is therefore the pressure $p$ that describes the physical nature of the "stuff" in the Universe. Conversely, if we measure the evolution of the Universe, which corresponds to measuring $a(t)$, then we can infer the evolution of $\rho$ and thus the evolution of $p$.

Usually we do not work with $p$ directly but instead we parameterize it with an "equation of state" parameter $w$ through $p=w \rho$. Now the physical nature is given by $w$, and some simple examples are:

$$
w=\left\{\begin{aligned}
1 / 3 \text { radiation } / \text { relativistic particles } \\
0 \text { dust (matter } / \text { nonrelativistic particles) } \\
-1 \text { cosmological constant. }
\end{aligned}\right.
$$

If we therefore found that $p=\rho / 3 \Leftrightarrow w=1 / 3$ then we could conclude that the Universe is filled with a gas of radiation. 


\begin{tabular}{llcc} 
Model & equation of state & $\begin{array}{c}\text { Dark sector } \\
\text { parameters }\end{array}$ & $\chi_{\text {min }}^{2}$ \\
\hline Constant $w$ & $w=w_{0}$ & 1 & 391.3 \\
Linear $(\mathrm{CPL})$ & $w=w_{0}+(1-a) w_{a}$ & 2 & 312.1 \\
Quadratic & $w=w_{0}+w_{1}(1-a)+w_{2}(1-a)^{2}$ & 3 & 309.8 \\
Cubic & $w=w_{0}+w_{1}(1-a)+w_{2}(1-a)^{2}+w_{3}(1-a)^{3}$ & 4 & 309.6 \\
$\Lambda \mathrm{CDM}$ & $w=-\left(1-\Omega_{m}\right) /\left[\Omega_{m} a^{-3}+\left(1-\Omega_{m}\right)\right]$ & 1 & 311.9 \\
\hline
\end{tabular}

Table 1. Number of parameters of the total dark sector equation of state (i.e. dark matter and dark energy) and best-fit chi-squared for various parameterizations of $w$, for background-only data (SN-Ia, CMB peak position, BAO and $H_{0}$, see discussion in section 3.2). The constant equation of state is ruled out while all others, including the $\Lambda \mathrm{CDM}$ equation of state, fit this data similarly well.

What constraints on $w$ do we get from cosmological data? The basic approach is simple: we just select a function $w(a)$ and compute $H(a)$ with the help of the Friedmann equation (3.4) and the conservation equation (3.5). Based on $H(a)$ we can then compute predictions for cosmological measurements that involve only the background geometry like luminosity and angular diameter distances. We can then use for example a Markov-Chain Monte Carlo (MCMC) method to evaluate the goodness of fit of the predictions with a likelihood function that is based on observations of these distances (see section 4.5 for an explanation of the MCMC method). In principle this is straightforward, but there are some technical questions. One important question is how to choose the function $w(a)$. There are many different ways to parameterize the equation of state parameter, for example as a function of redshift $z$ [4446] or as a function of scale factor $a$ [47-50]. Although in principle both descriptions are equivalent, one has to be careful to keep the parameterization well behaved to $z \gtrsim 1000$ when using high-redshift data, e.g. from the CMB [51]. This is less of a problem with functions of the scale factor, since it varies only in the interval $a \in[0,1]$, see [52] for a discussion of different parameterizations and potential problems. An elegant approach is given by the Principal Component Analysis (PCA), pioneered in a cosmological context by [53]. In PCA the function is expanded in a set of basis functions (for example in bins in $a$, but any functional basis will do) and the covariance matrix of the coefficients is diagonalized. The eigenfunctions then provide uncorrelated parameterizations of $w$, with the eigenvalues giving the precision with which those functions of $w$ can be measured. The drawback of PCA is that it depends on the data set used. Yet another possibility that has been investigated more recently is to use a Gaussian process for modeling $w(z)$ [54]. For now we simply use a Taylor expansion of $w$ in $a$ and increase the order until the goodness of fit no longer increases significantly. ${ }^{4}$

Another question is what data to use. Here one needs to be careful to avoid data sets that require the calculation of perturbations, the necessary formalism for that is discussed in the following section. In [58] we used type-Ia supernova data (SN-Ia, we used the Union sample [59]), the peak position in the CMB [60] and Baryonic Acoustic Oscillations (BAO, [61]), as well as the SHOES measurement of $H_{0}$ [62]. We then found the best-fit $\chi^{2}$ values listed in Table 1 (setting the spatial curvature to zero). For comparison we also list $\Lambda$ CDM which uses the single parameter $\Omega_{m}$. For this data, a quadratic expansion of $w(a)$ appears sufficient, we plot in Fig. 1 a random sample of such quadratic curves to given an idea of

\footnotetext{
${ }^{4}$ An alternative approach to modeling the evolution of $w$ parameterizes instead the expansion rate $H$ or the dark energy density $\rho_{\mathrm{DE}}[55-57]$.
} 
the range of allowed values of $w$. Although $\Lambda$ CDM provides a slightly worse fit than the quadratic model, it has fewer parameters and so passes this test. Only a constant total equation of state is really ruled out - we remind the reader that we consider here the total dark sector equation of state (i.e. dark energy and dark matter together) since the Einstein equations relate the geometry to the total energy-momentum tensor. We show in Fig. 1 a random sample of the best fitting quadratic $w(a)$ curves. At $a \lesssim 10^{-4}$ the Universe is radiation dominated which implies $w=1 / 3$, but the curves in the figure start later, during matter domination, and we can see that indeed $p \approx 0$ as expected for a Universe filled with pressureless dust. But already at relatively high redshift $w$ begins to decrease, and at $a \approx 0.5$ the expansion starts to accelerate as $w$ drops below $-1 / 3$. Today we have that $w \approx-0.8$, but with a large spread, mainly for two reasons: there is little data at very low redshifts (where anyway the local dynamics starts to be important), and $w$ affects the distances only through a double integration which smoothes its impact strongly.

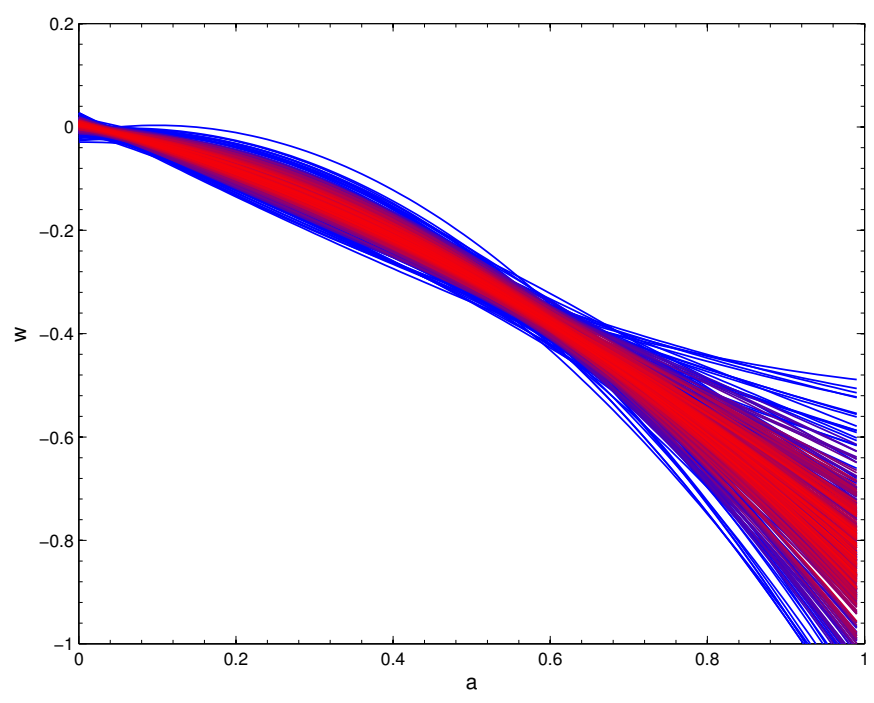

Figure 1. An illustration of the form of the best fitting quadratic $w(a)$ curves. A sparse sampling of 400 chain elements, colour coded by likelihood with the red (lighter) shading the highest, is shown. (Figure from [58])

An additional subtlety here is that we are usually not interested in the total pressure $p$, but instead in the pressure of the different constituents of the Universe. If for example the Universe was composed of matter and a cosmological constant, then we would find a total $w$ somewhere between 0 and -1 , see the last row of table 1 for the equation of $w$ in $\Lambda$ CDM. Instead, we would like to find two components, one with $w=0$ and a second one with $w=-1$. As we will discuss in more detail in section 5.1 this is in general not possible as the Einstein equations only link the geometry with the total energy momentum tensor (EMT). For the time being we will assume that the dark matter itself has been observed so that we can separate the total EMT into several components,

$$
T_{\mu \nu}^{(\text {tot })}=T_{\mu \nu}^{(\text {radiation })}+T_{\mu \nu}^{(\text {baryons })}+T_{\mu \nu}^{(\mathrm{DM})}+T_{\mu \nu}^{(\mathrm{DE})} .
$$

We put both electromagnetic radiation and neutrinos into the "radiation" EMT as we are not strongly interested in these components here. DM denotes the non-baryonic dark matter, 
and DE whatever it is that is responsible for the observed accelerated expansion of the Universe (including modifications of gravity). We are then interested in the equation of state parameter $w_{\mathrm{DE}}$ that characterizes the pressure of the DE component. We will discuss constraints on $w_{\mathrm{DE}}(a)$ in section 4.5 in the context of a canonical scalar field model for which the two contributions can be separated.

But even when we have measured $w_{\mathrm{DE}}(a)$ we will in general still not know what exactly the dark energy is, except maybe if the result matches one of the simple cases given in Eq. (3.6). In general there are many different possibilities that can lead to the same $w$. For example, a time-evolving $w$ could be due to a scalar field as well as a modification of gravity. Even if, as is indeed the case, observations are consistent with $w=-1$, we are still in trouble: we would like to interpret this result as a sign that the dark energy is a cosmological constant, but for the reasons discussed earlier (and elsewhere in this volume), we think that the cosmological constant is a problematic explanation for the dark energy.

Hence we would like to learn more about the nature of the dark energy. This is possible by exploiting the extra information encoded in the evolution of the perturbations in the Universe as we will discuss next. In addition, whenever we use data that requires calculating the evolution of perturbations (like e.g. the CMB, the full galaxy distribution or weak lensing) then we must provide a model that fixes the perturbation evolution. There is no "model independent way" to just use $w$ in that case. We either need to pick an explicit physical model or, as we will see in a moment, we need to choose precisely two extra functions to fix the evolution of the scalar perturbations to linear order. Whenever we think that we can get away by using only $w$, or maybe $w$ and the growth rate of matter perturbations, then we fix one or two other quantities to some value (and in the case of multiple data sets possibly to several different, incompatible values).

\subsection{Perturbation equations}

I will not enter into the details of cosmological perturbation theory since there are many excellent texts on the topic, for example [63-67]. Here we will work exclusively in the longitudinal (or conformal Newtonian) gauge. While the background quantities like $\rho, p$ and $H$ are only functions of time or of the scale factor, the perturbations are functions of both space and time. We will work in Fourier space as the linear perturbation equations are particularly simple there since all $k$-modes evolve independently. The perturbations themselves are random variables (so that in general we are really interested in the power spectra), and due to statistical isotropy and homogeneity they are functions of $k=|\mathbf{k}|$ (and of time) only, and independent of direction. We will further only consider scalar-type perturbations and assume that vector and tensor-type perturbations can be neglected (which may not always be the case).

The conservation equations for a fluid with equation of state parameter $w=p / \rho$ are then [65]

$$
\begin{aligned}
\delta^{\prime} & =3(1+w) \phi^{\prime}-\frac{V}{H a^{2}}-3 \frac{1}{a}\left(\frac{\delta p}{\rho}-w \delta\right) \\
V^{\prime} & =-(1-3 w) \frac{V}{a}+\frac{k^{2}}{H a^{2}} \frac{\delta p}{\rho}+(1+w) \frac{k^{2}}{H a^{2}} \psi-(1+w) \frac{k^{2}}{H a^{2}} \sigma
\end{aligned}
$$

where $\delta=\delta \rho / \rho$ is the density contrast, and we use $V=i k_{j} T_{0}^{j} / \rho$ as the scalar velocity perturbation (the reason for this choice will become clear in section 4.3). A prime designates 
a derivative with respect to the scale factor $a$. The right-hand side of the conservation equations also contains the pressure perturbation $\delta p(\sim$ the perturbation of the diagonal part of the space-space part of the EMT) and the anisotropic stress $\sigma(\sim$ the perturbation of the off-diagonal part of the space-space part of the EMT). These four variables $(\delta \rho, V, \delta p$ and $\sigma$ ) fully parameterize the scalar first-order perturbations of a general energy momentum tensor. The conservation equations need to be complemented by the Einstein equations. One of them gives the $\phi$ potential in terms of the fluid content in a form reminiscent of the Newtonian Poisson equation,

$$
k^{2} \phi=-4 \pi G_{N} a^{2} \sum_{\alpha} \rho_{\alpha}\left(\delta_{\alpha}+\frac{3 a H}{k^{2}} V_{\alpha}\right)=-4 \pi G_{N} a^{2} \sum_{\alpha} \rho_{\alpha} \Delta_{\alpha} .
$$

The sum over $\alpha$ on the right hand side runs over all fluids, and $\Delta$ is the comoving density contrast (while $\delta$ is the density contrast in the longitudinal gauge). The second potential is then related to the first through the anisotropic stresses present in the fluids,

$$
k^{2}(\phi-\psi)=12 \pi G_{N} a^{2} \sum_{\alpha}\left(1+w_{\alpha}\right) \rho_{\alpha} \sigma_{\alpha} .
$$

We notice that we have (at the perturbation level, i.e. without counting the background quantities) two functions that determine the (scalar part of the) metric, $\phi$ and $\psi$, and four functions that describe the behavior of a fluid and that define the (scalar part of the) energymoment tensor, $\delta, \delta p, V$ and $\sigma$. Hence in total we have six functions, and four equations, so that two of the functions cannot be determined. Given the structure of the perturbation equations where the two conservation equations describe the evolution of $\delta$ and $V$, and the two Einstein equations determine $\phi$ and $\psi$, it makes sense to consider $\delta p$ and $\sigma$ as the two free functions that describe the type of fluid present in the Universe (although this is in principle a choice to be made). This is very similar to the background case where the evolution of $\rho$ was determined by the conservation equation and $H$ was given by the Einstein equation, while we considered $p$ (or $w$ ) as a free function describing the nature of the fluid.

Is it possible to construct a quantity like $w$ that is of order unity and easier to interpret than $p$, to describe $\delta p$ ? For this purpose one often defines a rest-frame sound speed $c_{s}^{2}$, defined through $\delta p=c_{s}^{2} \delta \rho$ for the comoving pressure and density perturbation. In terms of the quantities in the conformal Newtonian gauge used here, this relation becomes

$$
\delta p=c_{s}^{2} \rho \delta+\frac{3 a H\left(c_{s}^{2}-c_{a}^{2}\right)}{k^{2}} \rho V
$$

where $c_{s}^{2}$ is still the rest-frame sound speed, and where $c_{a}^{2} \equiv \dot{p} / \dot{\rho}$ is called the adiabatic sound speed of the fluid. A fluid that has no internal degrees of freedom would have $c_{a}^{2}=c_{s}^{2}$, but in general such fluids are not viable dark energy models unless they mimic $\Lambda$ CDM very closely due to the perturbation evolution $[68,69]$. Models with internal degrees of freedom like scalar field models (where both the field and its time derivative can be seen as different degrees of freedom since the field obeys a second order equation of motion) have in general $c_{s}^{2} \neq c_{a}^{2}$. We will see in section 4.4, where we discuss the evolution of the perturbations in a generalized Quintessence model, that the role of the rest-frame sound speed is really to describe pressure support, i.e. it defines the existence of a sound horizon below which the density perturbations do not grow. The sound speed here is not necessarily related to the actual propagation velocity of perturbations, although the physics of pressure support is 
usually related to the speed with which perturbations can adjust and thus the sound speed coincides with the propagation velocity for many models, most notably for the Quintessence and K-essence models that we will discuss below. The sound speed does also not always provide a simpler description than $\delta p$, for an explicit example where this is not the case see the Quintom model in section 4.3 below.

Finally, as already discussed in general terms in the introduction, the energy momentum tensor considered here may be wholly or partially an effective, fictitious energy moment tensor. In this case also the fluid quantities $\delta p$ and $\sigma$ will be effective quantities. (In these cases as well we should not expect a simple $c_{s}^{2}$.) Nonetheless, we still can use the same formalism as these functions provide a general and complete description of first order perturbation theory around a FLRW metric. And as we will discuss in section 4.6 especially the presence of a non-zero anisotropic stress at late times (where contributions from relativistic particles to the total EMT are small) is a good indicator that we are dealing with a modified gravity model.

\subsection{Different descriptions}

As we have seen, one way to describe the dark energy component is through the entries in the effective energy moment tensor. But it is also possible to stay purely on the geometric side, and to parameterize just the metric degrees of freedom. In this case, we describe the evolution of the Universe through the evolution of the scale factor, or more often through the Hubble parameter $H(a)$, as well as through the two gravitational potentials $\phi(k, a)$ and $\psi(k, a)$. This is a description that it is quite close to the observations. A closely related parameterization uses not the two metric potentials but instead two linear combinations: $\Phi=\phi+\psi$ is the Weyl potential (its line of sight integral is the lensing potential that describes the deflection of relativistic particles or radiation due to gravity) as well as $\Pi=\phi-\psi$, the gravitational slip. Obviously the pairs $\{\phi, \psi\}$ and $\{\Phi, \Pi\}$ provide an equivalent description of the perturbative degrees of freedom. We should add here a health warning: there are different conventions in the literature for the signs of the gravitational potentials as well as for which one is the time-time and which one the space-space potential, so it is always a good idea to explicitly quote the metric used - my choice is given in Eq. (3.1), and I hope that I did not mix up conventions too often. Also the notation for the anisotropic stresses is not uniform. Here I call $\sigma$ the fluid anisotropic stress in the energy momentum tensor (with the appropriate pre-factors) and use $\Pi$ to denote the gravitational slip given by the difference of the two gravitational potentials, which is the geometric quantity that is linked directly to the fluid anisotropic stresses, see Eq. (3.11).

But is the description in terms of the metric potentials equivalent to the description in terms of the fluid variables $\delta p$ and $\sigma$ ? In the direction fluid variables $\rightarrow$ metric potentials it is easy to see that the answer is yes: given the fluid variables, we can use the Einstein equations and the conservation equations to compute the evolution of the metric potentials. In the opposite direction, it is less obvious. But since the Einstein equations (3.2) just identify, up to a coupling constant, the Einstein tensor (which is a function of the metric d.o.f.) with the EMT, we can project out the effective fluid quantities $\delta p$ and $\sigma$ also directly in the Einstein tensor and see what functions of the metric we recover. Doing this [70] we find that the geometric anisotropic stress is indeed determined by $\Pi=\phi-\psi$ (effectively just Eq. (3.11)), and that

$$
\delta p_{G}=\frac{1}{4 \pi G_{N}}\left[\ddot{\phi}+H(3 \dot{\phi}+\dot{\psi})-3 w_{G} H^{2} \psi-\frac{1}{3} k^{2} \Pi\right] .
$$


Here we also used the total "geometric" equation of state parameter

$$
w_{G}=-1-\frac{2}{3} \frac{\dot{H}}{H^{2}}
$$

If we use this pressure perturbation together with the anisotropic stress inferred from the difference of the potentials and the background evolution given by $w_{G}$ then we find again the same evolution of the metric potentials. The equivalence holds thus in both directions.

It is also possible to parameterize the geometric degrees of freedom through dimensionless variables that link two perturbative quantities, similarly to the way $w_{G} \operatorname{links} p$ and $\rho$. One example [71] is given by the pair $\{Q, \eta\}$ defined through

$$
\begin{aligned}
k^{2} \phi(k, a) & =-4 \pi G_{N} a^{2} Q(k, a) \rho_{m}(a) \Delta_{m}(k, a) \\
\psi(k, a) & =[1+\eta(k, a)] \phi(k, a)
\end{aligned}
$$

In this way, $Q$ parameterizes both a deviation from the Poisson equation due to a modification of GR and any extra contributions from perturbations beyond those of matter ${ }^{5}$. The latter is the case for example if we have a Quintessence-like model (see section 4.1) for which the dark energy perturbations are non-zero and will lead to $Q \neq 1$. The variable $Q$ can also model a variation of the gravitational constant $G_{N}$. Of course, in order to be general, both $Q$ and $\eta$ need to be functions of scale and time. This leads to an additional complication, namely that a multiplication in Fourier space corresponds to a convolution in real space, and vice versa. The conventional choice when limiting ourselves to first order perturbations is to define $Q$ as a multiplicative factor in $k$ space where the equations are easier to deal with as the modes decouple.

The choice of $Q$ and $\eta$ is not unique at all. The common theme is that we need to choose two non-degenerate functions to parameterize the extra perturbations. One often defines a parameter $\Sigma$ that is relevant for lensing through $\Sigma=Q(1+\eta / 2)$ so that the lensing potential is given by

$$
k^{2} \Phi=k^{2}(\phi+\psi)=-8 \pi G a^{2} \Sigma \rho_{m} \Delta_{m} .
$$

Another often used parameter is $\mu=(1+\eta) Q$ which quantifies the impact of the dark energy perturbations on a Poisson equation in $\psi$ in the same way as $Q$ for $\phi$. A compilation of different conventions can for example be found in [72], see also [73-75] for other discussions.

For use in data analysis, some combinations may be better suited than others because they may be more or less correlated. For example [76, 77] found that $\{\mu, \Sigma\}$ is a good choice for weak lensing, CMB and large scale structure data. We have also left out the question of how to parameterize the time and scale dependence of these functions. An early example is the Parameterized Post Friedmann framework [78] (with an even earlier example, pre-dating dark energy, in [79]), while the papers listed above provide further possibilities. The most general and flexible approach is probably the principal component analysis (PCA) approach already mentioned in the context of parameterizing $w$. In PCA the two functions are specified in a number of bins in $k$ and $a$, and the resulting covariance matrix between the bins is then diagonalized to provide uncorrelated measurements of the resulting eigenfunctions.

\footnotetext{
${ }^{5}$ Here we mean dark energy perturbations, i.e. we implicitly assume that the parameterization is used only at late times. At early times the radiation perturbations may be important, in which case they should probably be included explicitly.
} 


\subsection{Schematic Measurements}

Having discussed the freedom available, we also have to worry whether the free functions can be measured at all in cosmology. The aim here is not to discuss in detail the different observations. The goal of this short section is merely to check whether it is possible to measure the gravitational potentials in principle. Even this apparently straightforward question contains many different pitfalls. For example, dark energy might couple directly to baryons and light, and possibly do so in different ways. In this case we are going to reconstruct a metric that is not actually the metric we wanted to reconstruct. On the other hand, we could have transformed the action so that at least e.g. baryons are not coupled, and in general we assume that we did this. We will also assume that the constraints from direct tests for fifth forces affecting photons and baryons are applicable also on the scales of interest and limit such couplings to a level where they are not relevant.

If we are able to use the propagation of light and of baryons, then a possible high-level scheme can proceed as follows: the propagation of light is governed by the perpendicular derivative of the lensing potential $\phi+\psi$, so that with the help of weak lensing we are able to constrain this combination of the potentials (this is also the reason why the $\Sigma$ parameter is a good choice when using weak lensing data). Non-relativistic particles on the other hand are accelerated by $\nabla \psi$ (the contribution from $\phi$ is suppressed by the particle velocity). Observing the peculiar motion of objects on large scales, where they are not affected by gas-physics, it is thus possible to constrain $\psi$. One possibility is to use redshift space distortions on large scales for this purpose. In this way we can at least in principle obtain both $\phi$ and $\psi$ from the observations. Once we have measured both potentials independently, then we can e.g. read off whether the anisotropic stress is zero or not, since $\sigma$ is directly related to the gravitational slip $\Pi=\phi-\psi$ through Eq. (3.11).

We have not studied the measurements in any detail, but based on the above discussion we can conclude that a combined large-scale structure and weak-lensing survey can in principle constrain separately the two perturbation variables that characterize the dark sector. This means that if we include these variables in the likelihood of the observations, then we expect to obtain some constraints. How strong the constraints will be can be studied for example with the help of the PCA approach mentioned in the last section.

Here we have not yet used galaxy clustering. The reason is that in modified gravity models it is non-trivial to determine the bias between the clustering of galaxies and the clustering of the dark matter (e.g. [70]). However, as we have seen, we do not need this measurement at least in principle.

\section{$4 \quad$ Dark energy models}

\subsection{Quintessence}

Let us consider how the basic canonical scalar field model looks like in the phenomenological description discussed here. The action of the canonical scalar field (often called Quintessence or cosmon in a dark energy context [80-82]) $\varphi$ is given by

$$
S=\int d^{4} x \sqrt{-g}\left(-\frac{1}{2} \partial_{\mu} \varphi \partial^{\mu} \varphi-V(\varphi)\right) .
$$

From this action we can compute the evolution equation of $\varphi$ through variation with respect to the field, and the energy momentum tensor through variation with respect to the metric 
(if we added also the Einstein-Hilbert action then the variation wrt the metric would give the Einstein equation). We usually split the field into a homogeneous background field (a kind of condensate) and perturbations, $\varphi(k, t)=\bar{\varphi}(t)+\delta \varphi(k, t)$, and linearize the equations in the latter.

For the background quantities, one obtains

$$
\ddot{\bar{\varphi}}+3 H \dot{\bar{\varphi}}+\frac{d V}{d \varphi}=0, \quad \rho=\frac{1}{2} \dot{\bar{\varphi}}^{2}+V(\bar{\varphi}), \quad p=\frac{1}{2} \dot{\bar{\varphi}}^{2}-V(\bar{\varphi}) .
$$

We see the energy density and pressure depend on the potential and the evolution of the field, so a Quintessence field can dynamically change $w$. But we also find that $\rho+p=(1+w) \rho=$ $\dot{\bar{\varphi}}^{2} \geq 0$, implying that $w \geq-1$ as long as $\rho>0$.

It is furthermore easy to show that the evolution equation for $\bar{\varphi}$ together with the expressions for $\rho$ and $p$ is exactly the conservation equation $\dot{\rho}+3 H(\rho+p)=0$. The analogous computation can be done with the equations for the perturbations $\delta \varphi$, and one finds that they correspond exactly to the fluid perturbation equations in section 3.3 for a pressure perturbation defined through (3.12) with $c_{s}^{2}=1$ and no anisotropic stress, $\sigma=0[66,83]$. To first order in perturbation theory, a canonical scalar field is therefore behaving just like a fluid with speed of sound equal to the speed of light, and instead of evolving the Klein-Gordon equation of the field, we can just use the fluid perturbation equations.

There are however many aspects that are not captured by the fluid description of Quintessence. For example, the formalism gives no indication what equation of state $w$ to use. We would expect in general the physics to specify a scalar field potential, which then allows to integrate the evolution equation which in turn tells us what $w$ is. Of course this can be reversed: given a set of data, we can use the fluid description to constrain $w$, which in turn places constraints on the allowed shape of $V$ [84-86]. Another important point that is missing from the fluid description is the existence of fixed points and attractors in the evolution of $\varphi$, especially in the presence of other fluids. Scalar field models with exponential potentials are for example able to adjust their equation of state to follow the background evolution at a fixed fraction of the energy density. This allows for a partial solution to the coincidence problem: The scalar field starts out with a fairly arbitrary energy density early on, which then either decays rapidly or freezes until the scalar field energy density roughly matches the radiation energy density. The scalar field then "follows" radiation and latter matter. In this way it is natural that the scalar field today has an energy density comparable to the one in matter. Unfortunately it has proven very difficult to engineer a natural exit from this scaling phase, in general one needs to place a feature into the potential just at the right point. A very nice discussion of the scalar field evolution described as a dynamical system with a discussion of scaling properties for different potentials can be found for example in [7].

\subsection{Generalizing Quintessence}

As we have seen, the fluid picture of Quintessence is determined through the parameter choices $\left\{w, c_{s}^{2}=1, \sigma=0\right\}$, i.e. $w$ is a free function, but the pressure perturbation and the anisotropic stress are fixed. In this and the following section, we will see whether we can relax the two conditions, and what the implications of such generalizations are.

A simple way to change the sound speed is to allow a different kinetic term in the action (4.1). Writing $X \equiv-\frac{1}{2} \partial_{\mu} \varphi \partial^{\mu} \varphi$ the so-called K-essence action then becomes [87, 88]

$$
S=\int d^{4} x \sqrt{-g} K(X, \varphi),
$$


where $K(X)$ is a function of the standard kinetic term $X$, and we recover standard Quintessence through $K(X, \varphi)=X-V(\varphi)$. As for Quintessence, there is a vast literature on K-essence models, here I only summarize some standard results (see e.g. [13]). As usual through the variation of the action we can find the equation of motion and the energy momentum tensor. One finds that at the background level the energy density and pressure are given by

$$
p=K, \quad \rho=2 X K_{, X}-K
$$

where we dropped the dependence of $K$ on $X$ and $\varphi$ and where we used the shortened notation $K_{, X}=d K / d X$. The equation of state is therefore

$$
w=\frac{p}{\rho}=\frac{K}{2 X K_{, X}-K}
$$

which for Quintessence (where $K_{, X}=1$ ) coincides with with the equation of state derived from Eq. (4.2). When studying the perturbations, one finds that also K-essence models are described by a sound speed given by

$$
c_{s}^{2}=\frac{p_{, X}}{\rho_{, X}}=\frac{K_{, X}}{2 X K_{, X X}+K_{, X}}
$$

and vanishing anisotropic stress, $\sigma=0$. K-essence models therefore generalize the Quintessence models to $\left\{w, c_{s}^{2}, \sigma=0\right\}$, where now $w$ and $c_{s}^{2}$ are free. The sound speed in K-essence models is no longer equal to the speed of light. It can be larger or smaller than 1 and also vary over time. We note that for the Quintessence case we recover again the expected result $c_{s}^{2}=1$, while for the class of models with $K(X)=X^{\alpha}-V(\varphi)$ we have $c_{s}^{2}=1 /(2 \alpha-1)$, i.e. we can choose a constant sound speed through the choice of $\alpha$.

An important point (and a main motivation for introducing K-essence) that is not apparent in a fluid formulation, is that in K-essence models the evolution of the homogeneous "background" field can be made to depend on the overall expansion rate of the Universe so that the onset of matter dominated expansion triggers a transition to a later accelerated expansion stage dominated by the K-essence field. That is, K-essence models can (for suitable choices of the kinetic function) overcome the coincidence problem of the cosmological constant by linking the onset of dark energy domination to the earlier onset of matter domination. As matter perturbations do not grow during radiation domination, structure and therefore intelligent lifeforms similar to us can also form only after the radiation-matter transition and so a coincidence between the emergence of species observing the cosmos and the transition to dark energy dominated expansion is expected in such models. However, a drawback of these models is that the speed of sound necessarily becomes larger than unity in all models that can solve the coincidence problem [89]. Whether such a superluminal propagation of information is problematic is a subject of debate [90, 91]: in general if information can be transmitted faster than light for all observers, then it is possible to construct closed time-like curves and so to transmit information into the past. This leads to obvious problems with causality. On other hand, if information travels only faster than $c$ with respect to some observers, for example with respect to the rest-frame of the K-essence fluids on which the perturbations propagate, then causality problems can be avoided but Lorenz invariance is broken and we have introduced an effective aether through the presence of the K-essence condensate. 


\subsection{Phantom crossing}

As seen in section 4.1, a canonical scalar field model cannot cross the "phantom divide" $w=-1$. This does not mean however that such a crossing is impossible: we will briefly discuss a two-fluid example here, and another possibility is mentioned in section 5.1, namely that coupled dark matter - dark energy models can lead to apparent phantom crossing. In addition, theories where GR is modified can in general cross as well, since there is no "real" dark energy field present. When analyzing data, and especially since the data indicates that $w \approx-1$, it is important to not exclude the possibility $w<-1$ by construction. In this section we will review at a purely classical level the behavior of the perturbations close to $w=-1$ and how to avoid problems when $w$ crosses -1 (at the quantum level phantom fields would allow for spontaneous vacuum decay, but potentially viable models could still be constructed, see e.g. [92, 93]). An additional motivation for this section is that it illustrates explicitly the limitations inherent in parametrizing the pressure perturbations purely in terms of a rest-frame sound speed: although this is often a good choice that significantly simplifies the description of the situation, this is not always the case.

When looking at the standard perturbation equations, as e.g. found in [65] where the velocity perturbations $\theta$ are defined through $T_{0, i}^{i} \propto(\rho+p) \theta$, one finds terms like

$$
\dot{\theta}=-\frac{\dot{w}}{1+w} \theta+\ldots
$$

The division by $1+w$ looks like a problem, as it would lead to a divergence in $\theta$ when trying to cross $w=-1$. But since $\rho+p$ was factored out in the definition of $\theta$, the energy-momentum tensor can stay finite even if $\theta$ diverges. Indeed, if the divergence of $T_{0 i}$ does not go to zero at crossing, then $\theta$ will necessarily diverge. But this is only an apparent problem and easily cured by changing slightly the definition of the the velocity perturbations, and it is the reason why we use here $V$ defined through $T_{0, i}^{i} \propto \rho V$.

A more severe problem appears when we look at the pressure perturbation parameterized through a sound speed in the rest-frame. The gauge transformation from the rest-frame to another frame involves the adiabatic sound speed $c_{a}^{2}=\dot{p} / \dot{\rho}$ which can also be written as

$$
c_{a}^{2}=w-\frac{\dot{w}}{3 H a(1+w)} .
$$

The adiabatic sound speed necessarily diverges at phantom crossing, except when crossing with $\dot{w}=0^{6}$. Since $\delta p$ appears directly in the EMT, its divergence implies a potential singularity in the metric and is not acceptable on physical grounds. The reason for this divergence is that the rest-frame of the dark energy is badly defined when $w=-1$, so we chose to fix the pressure perturbation to a finite value in the one gauge that we should not have used. Seen in this way, the problem becomes easy to remedy: we just have to keep $\delta p$ finite in another gauge, for example in the longitudinal gauge. Then nothing at all happens at phantom crossing. In practice, this is what we will do later on when using supernova and CMB data to put constraints on $w$, allowing also for $w<-1$. More precisely, we regularize the adiabatic sound speed by setting

$$
\tilde{c}_{a}^{2}=w-\frac{\dot{w}(1+w)}{3 \mathcal{H}\left[(1+w)^{2}+\lambda\right]}
$$

\footnotetext{
${ }^{6} \mathrm{~A}$ more detailed analysis shows that for $c_{s}^{2}=0$ the crossing is possible even if $\dot{w} \neq 0$ [83] - interestingly, this is the same condition as the one found in [94] with the help of effective field theory, and an explicit example can be found in [95].
} 
where $\lambda$ is a tunable parameter which determines how close to $w=-1$ the regularisation kicks in. A value of $\lambda \approx 1 / 1000$ appears to work reasonably well [83].

Finally, it is instructive to have a quick look at the behavior of the perturbations in the "Quintom" model of dark energy [96, 97]. This model consists of two scalar fields, one with a constant equation of state parameter $w_{1}>-1$ and another one with $w_{2}<-1$ (which necessitates changing the sign of the kinetic term, introducing a ghost degree of freedom, but here we are only concerned with the classical behavior of the perturbations at the linear level). As the ratio of the energy densities of the two fields scales as $\rho_{2} / \rho_{1}=a^{-3\left(w_{2}-w_{1}\right)}$ and since $w_{2}-w_{1}<0$ we see that the second field becomes more important over time, and so the total equation of state parameter will evolve from $w \approx w_{1}$ at early times towards $w \approx w_{2}$ at late times, and will cross $w=-1$ somewhere in between. Yet since we are just dealing with two scalar fields with constant $w$, we also know that nothing strange will happen to the perturbations. In this system, we can compute in detail all the perturbations and study their evolution [83]. The total pressure perturbation can be written in terms of the total effective quantities in the form

$$
\delta p_{\text {eff }}=\hat{c}_{s, \text { eff }}^{2} \delta \rho_{\text {eff }}+\delta p_{\text {rel }}+\delta p_{\text {nad }}+3 \mathcal{H}\left(\hat{c}_{s, \text { eff }}^{2}-c_{a}^{2}\right) \bar{\rho}_{\text {eff }} \frac{V_{\text {eff }}}{k^{2}}
$$

where $\hat{c}_{s, \text { eff }}^{2}=1$ since this is the sound speed of both fields, $\delta p_{\text {rel }}$ is the contribution from the relative density perturbation of the two fields (corresponding to a gauge invariant relative entropy perturbation) and $\delta p_{\text {nad }}$ a non-adiabatic contribution from the relative motion of the fields. We plot in the left panel of Fig. 2 the evolution of these terms as the total equation of state crosses $w=-1$. Each contribution individually diverges, but their sum remains finite and well behaved. If one now tries to extract a total effective sound speed through the usual formula

$$
\delta p_{\mathrm{eff}}=c_{x}^{2} \delta \rho_{\mathrm{eff}}+3 \mathcal{H}\left(c_{x}^{2}-c_{a}^{2}\right) \bar{\rho}_{\mathrm{eff}} \frac{V_{\mathrm{eff}}}{k^{2}} .
$$

then all the terms get mixed up, and the resulting pseudo-sound speed $c_{x}^{2}$ is plotted in the right-hand panel of Fig. 2. This pseudo-sound speed that is not connected to any physical quantity has acquired a scale-dependence and additionally diverges at $w=-1$.

There are two main points to take away from this section, apart from the phantomcrossing discussion. Firstly, although we have argued that the different parameterizations of the degrees of freedom available in the metric and in the energy momentum tensor are in principle all equivalent, we have been provided here with an explicit example that some parameterizations are nonetheless better than others, and that the answer to the question of which one is best depends on the situation. Usually, the rest-frame sound speed $c_{s}^{2}$ is a good parameter. It manages to capture the main characteristic of a canonical scalar field in a single number $\left(c_{s}^{2}=1\right)$ while a description in terms of $\delta p$ would look much more complicated, and in addition the parameter itself describes a physical object (the propagation velocity of the scalar field perturbations) of direct relevance to cosmology (as the propagation speed gives rise to a sound horizon). However, in a situation where the direct physical correspondence is lost, for example in the two-field Quintom model, the total "rest-frame" sound speed takes a very complicated form, with scale dependence and divergences. Naively, one would probably not have allowed such strange behavior, thinking it unphysical! For this reason, it appears important to use several parameterizations and to test with forecasts whether we expect to see interesting effects for the models where a given parameterization is simple.

But on the other hand, the phantom crossing example also shows how in some cases the phenomenological approach allows a straightforward generalization of the behavior of 

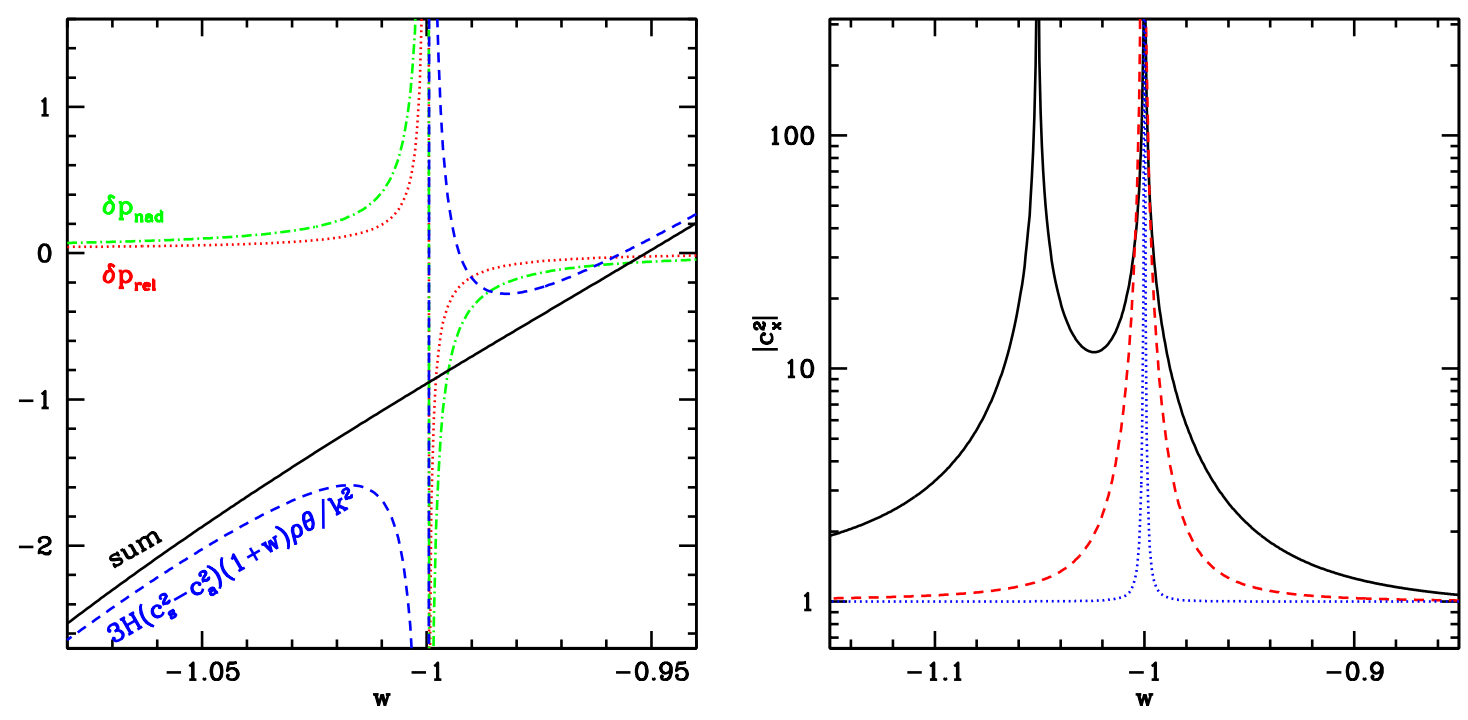

Figure 2. Left: This figure shows the different divergent contributions to the pressure perturbation, Eq. (4.10), multiplied by $10^{9}$. The relative pressure perturbation is shown as red dotted line, the non-adiabatic pressure perturbation as green dash-dotted line and the contribution from the gauge transformation to the conformal Newtonian frame as blue dashed line. Each of the contributions diverges at the phantom crossing, but their sum (shown as black solid line), and so $\delta p$, stays finite. Right: We plot the apparent sound speed $c_{x}^{2}$ defined by Eq. (4.11) for three different wave vectors, $k=1 / H_{0}$ (black solid line), $k=10 / H_{0}$ (red dashed line) and $100 / H_{0}$ (blue dotted line). Although the real sound speed is just $c_{s}^{2}=1$, the apparent sound speed is scale dependent, diverges at $w=-1$ and can even become negative. (Figure from [83])

the original model. A canonical scalar field model would never cross $w=-1$. There is no choice of the potential that can be made for which $w<-1$ (as long as the energy density is kept positive). If we just restricted ourselves to reconstructing Quintessence potentials, we would never even have realized that the possibility $w<-1$ exists. This is an example where the phenomenological approach allows to probe the available degrees of freedom in a more model-independent way: let us assume that the true model indeed has $w<-1$. Then, if all we knew were scalar fields, we would presumably keep finding that $\Lambda \mathrm{CDM}$ is the best fit, even though there are other models, that we did not think of, that would be better and could rule out $\Lambda \mathrm{CDM}$.

\subsection{Perturbation evolution in generalized Quintessence models}

Let us take a closer look at the evolution of perturbations in Quintessence-type models with vanishing anisotropic stress, but with an arbitrary constant sound speed $c_{s}^{2}$. In addition, we will assume that the equation of state parameter $w$ is constant. This is strong assumption (and precludes for example the possibility of early dark energy) but significantly simplifies the analysis in the fluid picture. In this case the perturbation equations (3.8) and (3.9) 
become

$$
\begin{aligned}
\delta^{\prime} & =-\frac{V}{H a^{2}}\left(1+\frac{9 a^{2} H^{2}\left(c_{s}^{2}-w\right)}{k^{2}}\right)-\frac{3}{a}\left(c_{s}^{2}-w\right) \delta+3(1+w) \phi^{\prime} \\
V^{\prime} & =-\left(1-3 c_{s}^{2}\right) \frac{V}{a}+\frac{k^{2} c_{s}^{2}}{H a^{2}} \delta+(1+w) \frac{k^{2}}{H a^{2}} \phi
\end{aligned}
$$

and we only have a single potential given by

$$
k^{2} \phi=-4 \pi G a^{2} \sum_{j} \rho_{j}\left(\delta_{j}+\frac{3 a H}{k^{2}} V_{j}\right)
$$

since $\psi=\phi$ due to the vanishing anisotropic stress. Following [98] we also assume matter domination so that the background evolution is given by

$$
H^{2}=H_{0}^{2} \Omega_{m} a^{-3} \text {. }
$$

For the matter dominated era, the (growing) solution of the matter perturbations $(w=\delta p=$ $0)$ is well known,

$$
\begin{aligned}
\delta_{m} & =\delta_{0}\left(a+3 \frac{H_{0}^{2} \Omega_{m}}{k^{2}}\right)=\delta_{0} a\left(1+3 \frac{H^{2} a^{2}}{k^{2}}\right) \\
V_{m} & =-\delta_{0} H_{0} \sqrt{\Omega_{m}} a^{1 / 2} \\
k^{2} \phi & =-\frac{3}{2} \delta_{0} H_{0}^{2} \Omega_{m}
\end{aligned}
$$

which can be checked easily by inserting the solution into the perturbation equations. Since we are working with linear perturbation equations, the overall scale is a free parameter, here called $\delta_{0}$. It is in general a function of $k$ and is set by initial conditions that should include the perturbation generation in the early Universe (inflation) and the subsequent evolution during radiation domination. We also observe that the gravitational potential is constant in time.

We can now study the scalar field perturbations without the need to look at the actual field evolution, since these equations are equivalent to the fluid equation with the appropriate value of the fluid parameters. We first look at perturbations larger than the sound horizon, $k \ll a H / c_{s}$. In this case, we neglect all terms containing the sound speed in Eq. (4.12), effectively setting $c_{s}^{2}=0$. The solution for the velocity perturbation is (neglecting a decaying solution $\propto 1 / a)$

$$
V=-\delta_{0}(1+w) H_{0} \sqrt{\Omega_{m}} a^{1 / 2} .
$$

Up to the prefactor $(1+w)$ this is the same as for the matter velocity perturbations. We find that this expression is valid on scales larger than the sound horizon even if the sound speed is non-zero.

It is now straightforward to insert this solution for the dark energy velocity perturbation into Eq. (4.12). Again setting $c_{s}^{2}=0$ we find the solution

$$
\delta=\delta_{0}(1+w)\left(\frac{a}{1-3 w}+\frac{3 H_{0}^{2} \Omega_{m}}{k^{2}}\right)
$$

where we neglected a term proportional to $a^{3 w}$ which is decaying as long as $w$ is negative. Not surprisingly, also this solution becomes equal to the one for matter perturbations for $w \rightarrow 0$. 
Relative to the matter perturbations the dark energy perturbations are suppressed by the factor $(1+w)$. This factor is necessarily always there, as the gravitational potential terms contain it. It can be thought of as modulating the strength of the coupling of the dark energy perturbations to the perturbations in the metric. For $w=-1$ the dark energy perturbations are completely decoupled (in the sense that they do not feel metric perturbations - but they can still produce them if the dark energy perturbations are not zero).

The most important feature of the scalar field perturbations compared to the matter perturbations is the existence of a sound horizon. Inside the light horizon, the dark matter perturbations grow linearly with $a$ (until the perturbations become non-linear). The dark energy perturbations on the other hand will eventually encounter their sound horizon if $c_{s}^{2}>0$. Once inside the sound horizon, they will stop growing. This means that the dark energy perturbation spectrum is cut off on small scales.

To get a solution on small scales, $k \gg a H / c_{s}$, we start again with the equation for the velocity perturbation. However, we expect the two terms with $k^{2}$ to cancel to a high degree to avoid large velocity perturbations, or in other words

$$
\delta=-\frac{(1+w) \phi}{c_{s}^{2}}=\frac{3}{2}(1+w) \frac{H_{0}^{2} \Omega_{m}}{c_{s}^{2} k^{2}} \delta_{0} .
$$

As expected the dark energy perturbations stop growing and become constant inside the sound horizon. The velocity perturbations are now given simply by using Eq. (4.12) and inserting Eq. (4.20):

$$
V=-3 H a\left(c_{s}^{2}-w\right) \delta=-\frac{9}{2}(1+w)\left(c_{s}^{2}-w\right) \frac{H_{0}^{3} \Omega_{m}^{3 / 2}}{c_{s}^{2} k^{2}} a^{-1 / 2} .
$$

The extra term in brackets in Eq. (4.12) is not important for the scales of interest here.

As the horizons grow over time, a fixed wave number $k$ will correspond to a scale that is larger than the light horizon, $k<a H$, at early times, and eventually it will enter the light horizon and later the sound horizon. This makes it possible to illustrate the behavior of the perturbations in the different regimes in a single figure: In the left panel of Fig. 3 we plot the numerical solution for the dark energy density contrast for $k=200 H_{0}$ as well as the expressions (4.19) and (4.20). It is easy to see how the perturbations start to grow inside the causal horizon but how the growth stops when the sound horizon is encountered and pressure support counteracts the gravitational collapse.

In the right-hand panel of Fig. 3 we show the size of $\delta_{\mathrm{DE}}$ relative to $\delta_{m}$. There are two effects: on large scales the dark energy perturbations are suppressed by a factor proportional to $(1+w)$ relative to the matter perturbations (and an additional factor $\sim 4$ inside the light horizon), and as there is no sound horizon for perfectly cold dark matter, $\delta_{m}$ continues to grow on small scales so that $\delta_{\mathrm{DE}} / \delta_{m} \propto 1 / a$ inside the dark energy sound horizon. If we want to express the impact of the dark energy perturbations on the gravitational potentials with the help of the $Q$ variable, as defined in (3.15), we should in principle consider the rest-frame density perturbations which is a negligible change on small scales and on large scales (and $w \approx-1)$ just gives $\Delta_{\mathrm{DE}} \approx \Delta_{m}(1+w) / 4$. Additionally we need to take into account the relative mean density, $\rho_{\mathrm{DE}} / \rho_{m}=\Omega_{\mathrm{DE}} / \Omega_{m} a^{-3 w}$ for constant $w$. The combination of these effects can then be expressed by the interpolating, approximate formula

$$
Q-1=\frac{1-\Omega_{m}}{\Omega_{m}}(1+w) \frac{a^{-3 w}}{1-3 w+\frac{2}{3} \nu(a)^{2}}
$$



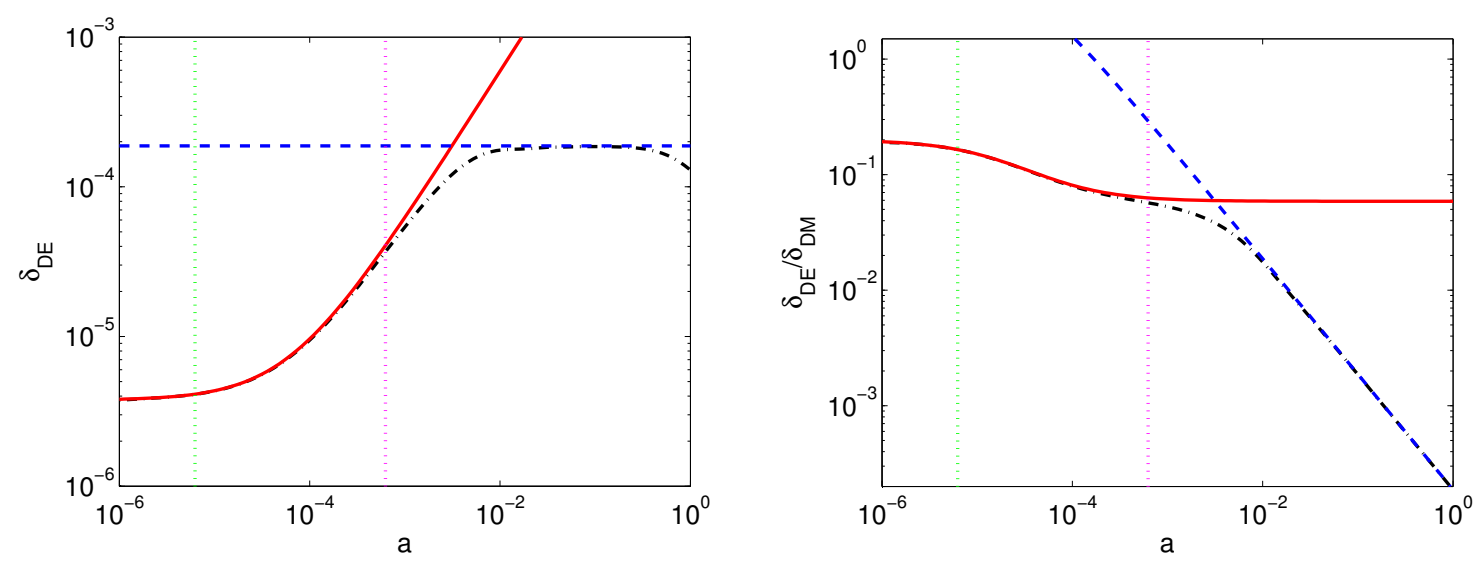

Figure 3. Left: The figure shows the behavior of the variable $\delta_{\mathrm{DE}}$ (in a universe without radiation). The black dot-dashed line is the numerical solution with $c_{s}^{2}=0.01$ and $w=-0.8$ for the mode $k=200 H_{0}$. The red solid line is the approximation on scales above the sound horizon, Eq. (4.19) and the blue dashed line is the approximation to the scales below the sound horizon, Eq. (4.20). The two vertical lines give the scale factor at which the mode enters the Hubble horizon (left line) and the sound horizon (right line). The numerical solution shows how the perturbations decay at late times when matter domination ends, but radiation was omitted from the numerical calculation to allow for a longer dynamic range in $a$ to illustrate the different regimes. Right: The ratio of dark energy to dark matter perturbations. For scales inside the dark energy sound horizon, the relative amplitude decreases linearly with $a$. We can also see that the ratio of the perturbations is described well by the fitting formula even when dark energy domination starts to affect the perturbation evolution.

where we introduced $\nu(a)^{2} \equiv k^{2} c_{s}^{2} a /\left(H_{0}^{2} \Omega_{m}\right)$ (the amount by which a mode is inside the sound horizon) and also assumed flatness so that $\Omega_{\mathrm{DE}}=1-\Omega_{m}$. This expression is quite good on scales much larger and smaller than the sound horizon, while close to the sound horizon it misses some transient effects. From this formula, we can see that today $(a=1)$ the impact of the dark energy perturbation on large scales $(\nu \ll 1)$ is of the order of $3(1+w) / 4$, while on small scales $(\nu \gg 1)$ it behaves roughly like $4(1+w) / \nu^{2}$. On scales above the sound horizon therefore the perturbations can contribute up to $10 \%$ to $\phi$ given today's limits on $w$, but inside the sound horizon the dark energy impact is strongly suppressed. Additionally, as we go back into the past, the dark energy contribution to $\phi$ scales like $a^{-3 w} \sim 1 / a^{3}$, so at a redshift of $z=1$ we get an additional suppression by a factor of 6 to 8 .

The direct detection of the perturbations is important because there is much more information on models encoded in the perturbation variables than in the evolution of $w$. Measuring the behavior of perturbations is akin to finding a fingerprint at a crime scene. By matching the perturbation fingerprint against model predictions we may be able to understand what the physics behind the accelerated expansion is. Unfortunately the smallness of the perturbations in Quintessence and K-essence type models make it very difficult to directly measure the perturbations. In [99] we found that even large future weak lensing and galaxy clustering observations can only hope to measure the sound speed (which we use as a proxy to decide whether it is possible to detect the perturbations) if $c_{s}^{2}<10^{-4}$, a conclusion also supported by other studies $[100,101]$.

Another point to take away from this section is how the use of the fluid equations and fluid variables (rather than, say, the potential $V(\phi)$ of a Quintessence model or the 
exponent $\alpha$ of a K-essence model with $K(X)=X^{\alpha}$ ) allowed us to study the behavior of the perturbations in way that is more abstract from the fundamental model point of view, but that emphasizes the physical evolution of the perturbations and allows to derive relatively simple yet quite accurate formulae in order to study the expected observationally relevant effects. The main effect for these models is the existence of a sound horizon within which the perturbation growth is suppressed by pressure support. Because of this sound horizon, only perturbations in sufficiently "cold" dark energy with $c_{s}^{2} \ll 1$ can be detected.

\subsection{Observational constraints on the Quintessence equation of state}

Already one of the original supernova papers included constraints on a constant equation of state parameter $w$ for the dark energy [5], an analysis that was extended in [102]. Later papers like [85] and [103] allowed for an evolving $w(a)$, parameterizing either the distance (for a fixed $\Omega_{m}$ ) or the Quintessence potential. These early analyses only considered distance data and neglected the evolution of the dark energy perturbations. For this reason they needed to either fix $\Omega_{m}$ or use a sufficiently stiff parameterization to break the dark degeneracy that we will discuss in section 5.1.

Considering the evolution of the dark energy perturbations is not only important in order to use the additional information in probes like the CMB, but also because all dark energy models apart from $\Lambda$ necessarily have perturbations, and as demonstrated explicitly in [104] they are important even for Quintessence models when using the full CMB data. In $[105,106]$ the perturbations were taken into account for a family of Quintessence potentials, and in $[51,107]$ we parameterized directly $w(a)$ and reconstructed explicitly a Quintessence potential for which the Klein-Gordon equation was solved. Since these papers used actual Quintessence models, they always set $c_{s}^{2}=1$, while for example [108] used directly the fluid equations and allowed for a free dark energy sound speed.

In this section we follow the approach of $[109,110]$, where the fluid perturbations (effectively (3.8) and (3.9) except that most Boltzmann codes work in the synchronous gauge rather than the longitudinal gauge) were inserted into a modified version of CMBFAST [111]. Differences are that we use a modified CAMB [112] instead of CMBFAST to compute the constraints shown in Fig. 4, and that we include additionally the phantom crossing prescription defined through the regularized adiabatic sound speed of Eq. (4.9).

Computing constraints on the equation of state parameter of the dark energy is a priori straightforward, as already outlined in section 3.2: we pick a model $\mathcal{M}$, here defined through a parameterization of $w(a)$ and a choice of $\delta p$ and $\sigma$ (we will call the set of parameters $\theta$, which is in general a vector of length $m$ for $m$ different parameters and includes also the cosmological parameters). We also choose some data $D$. For the purpose of the statistical analysis, the data is encoded in a likelihood, which is the probability density of observing the data for a given model and a given set of parameters, usually written as a function of the parameters $\mathcal{L}(\theta)=P(D \mid \theta, \mathcal{M})$. To give a simple example, if our model were a Gaussian probability distribution function (pdf) with unknown mean $\mu$ and known variance $\sigma^{2}$ and we tried to measure $\mu$ (so that $\theta=\mu$ ) from a set of data $D=\left\{x_{1}, x_{2}, \ldots, x_{n}\right\}$ drawn independently from the Gaussian pdf then the likelihood would be

$$
\begin{aligned}
\mathcal{L}(\mu) & =P\left(D \mid \mu, \sigma^{2}\right)=\prod_{i=1}^{n} \frac{1}{\sqrt{2 \pi \sigma^{2}}} \exp \left\{-\frac{1}{2} \frac{\left(\mu-x_{i}\right)^{2}}{\sigma^{2}}\right\} \\
& =\frac{1}{\left(2 \pi \sigma^{2}\right)^{n / 2}} \exp \left\{-\frac{1}{2} \sum_{i=1}^{n} \frac{\left(\mu-x_{i}\right)^{2}}{\sigma^{2}}\right\} \propto e^{-\chi^{2} / 2} \text { for } \chi^{2}=\sum_{i=1}^{n} \frac{\left(\mu-x_{i}\right)^{2}}{\sigma^{2}} .
\end{aligned}
$$


Traditional (frequentist) statistics works directly with the likelihood, while Bayesian statistics is interested in the probability of the parameters given the data, $P(\theta \mid D, \mathcal{M})$. With the help of Bayes theorem the two can be linked,

$$
P(\theta \mid D, \mathcal{M})=\frac{P(D \mid \theta, \mathcal{M}) P(\theta \mid \mathcal{M})}{P(D \mid \mathcal{M})} .
$$

The second term in the numerator, $P(\theta \mid \mathcal{M})$, is called the prior since it corresponds to the prior knowledge of how the parameter values are distributed, before the data is taken into account. The quantity in the denominator, $P(D \mid \mathcal{M})$ is independent of the parameters and thus just an irrelevant proportionality constant when trying to constrain the parameters. It is however an important quantity for model-comparison purposes in the Bayesian framework (e.g. [113$116])$. How to choose the prior is not an entirely simple question. For a parameter like the mean which can be at an arbitrary location, a natural choice is to choose $P(\mu \mid \mathcal{M})$ constant. On the other hand if we wanted to estimate the variance $\sigma^{2}$ that is rather independent of scale, a prior of the form $P(\sigma \mid \mathcal{M}) \propto 1 / \sigma$ is the usual choice. A detailed discussion of priors (and of Bayesian statistics in general) can be found in the book by Jaynes [117]. One of the standard works on classical statistics are the two volumes by Feller $[118,119]$.

Once we have chosen a likelihood we need to compute confidence intervals, i.e. find regions in parameter space that encompass a certain percentage (e.g. 95\%) of the probability. This requires effectively to compute an integral in a potentially high-dimensional parameter space. High-dimensional integrals are generally very difficult to compute numerically; for about a decade now the preferred solution in cosmology has been to use a Markov-Chain Monte Carlo (MCMC) method with Metropolis-Hastings acceptance criterion [120]. The algorithm is very simple:

1. Pick an initial point in parameter space, $\theta_{0}$, and evaluate the likelihood at that point, $\mathcal{L}_{0}=\mathcal{L}\left(\theta_{0}\right)$.

2. Choose a new point $\theta_{1}$ so that the probability of picking $\theta_{1}$ when at $\theta_{0}$ is the same as the probability of picking $\theta_{0}$ when at $\theta_{1}$. (This condition can be relaxed by allowing for a slightly more complicated acceptance criterion in step 4 below.)

3. Evaluate the likelihood at the new point, $\mathcal{L}_{1}=\mathcal{L}\left(\theta_{1}\right)$.

4. Accept the step with probability $P=\min \left(1, \mathcal{L}_{1} / \mathcal{L}_{0}\right)$. If the new point is accepted, set $\theta_{0}=\theta_{1}$ and $\mathcal{L}_{0}=\mathcal{L}_{1}$.

5. Record $\theta_{0}$ as a new element in the chain (even if the step was not accepted and $\theta_{0}$ has not changed!).

6. Go to step 2 and keep going.

The usual way to choose a parameter vector $\theta_{1}$ in step 2 above is by picking a random vector $\Delta \theta$ from a Gaussian pdf with zero mean and a given, fixed covariance matrix, and setting $\theta_{1}=\theta_{0}+\Delta \theta$. A good choice for the covariance matrix of the step-distribution is an approximation to the parameter covariance matrix (formally, when allowing for an infinite number of MCMC steps, it does not matter what step-distribution is chosen as long as it is symmetric and can reach all of parameter space, but in practice it is very important for the efficiency of the algorithm). One also needs to remove an initial non-stationary period of 
the MCMC evolution, the burn-in, and one needs to ensure that all the parameter space has been probed sufficiently, i.e. that the chain has converged. See e.g. [121] for more details on MCMC methods in cosmology.

The output of the MCMC method is a so-called chain of parameter values that provide a random sample from the posterior distribution of the parameters $\theta$. Since the density of points is proportional to the value of the likelihood, we can marginalize (integrate out) parameters by just ignoring them. To get the marginalized pdf of a given parameter $\theta_{i}$, we can just look at the histogram of the values of that parameter in the chain, while for two-dimensional confidence contours one needs to determine an area that contains e.g. $95 \%$ of the probability. This is typically done by discretizing the relevant $2 \mathrm{D}$ parameter sub-space onto a grid and associating to each grid-square the number of points in the chain that lie there. From the discretized 2D-histogram it is then straightforward to derive the desired contour. A popular package for cosmological MCMC applications, with likelihoods for many data sets and tools for post-processing chains, is $\operatorname{CosmoMC}^{7}[121,122]$. A good discussion of MCMC methods and extensions (and generally a lot of statistics) can be found in the book by MacKay $[123]^{8}$. There are also other numerical methods to infer parameter constraints and compute model probabilities. One approach that has become quite popular in cosmology over the last few years is nested sampling [52, 124-126], another one is population-Monte Carlo [127].
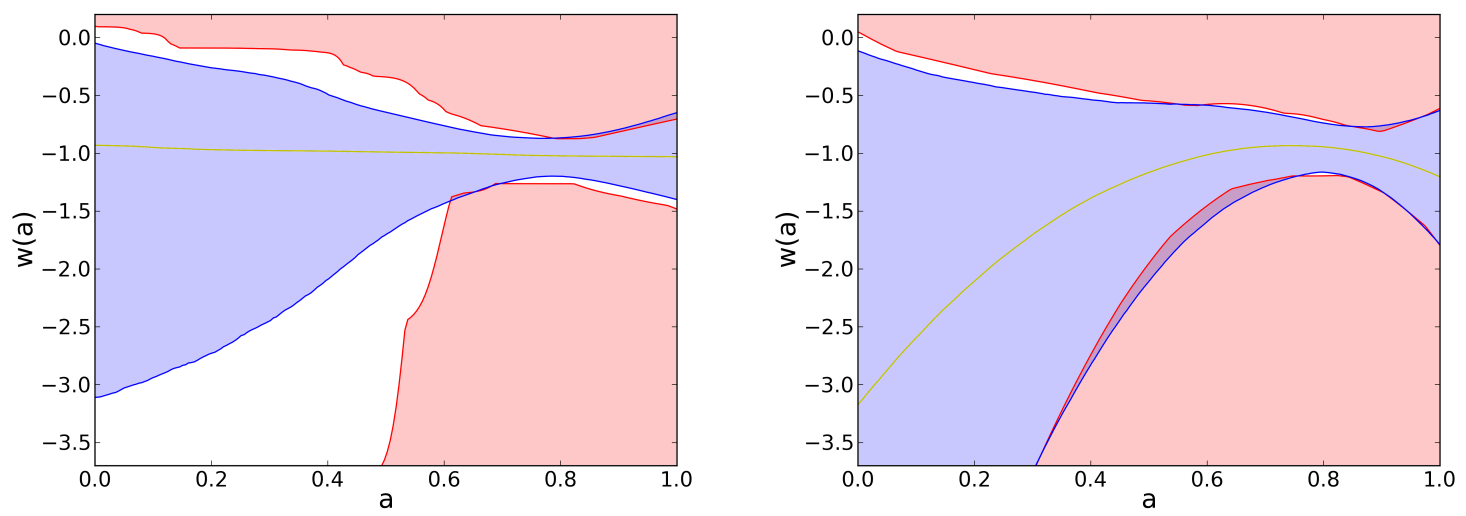

Figure 4. Constraints on two parameterizations of $w(a)$ for a Quintessence model $\left\{c_{s}^{2}=1, \sigma=\right.$ $0\}$ from WMAP CMB data and the Union type-Ia supernova data. The blue (inner) shaded area corresponds to the location of $95 \%$ of the accepted $w(a)$ 's, while any model in the red (outer) shaded area has an effective $\chi^{2}$ that is at least 4 worse than that of the best model. The left panel uses a kink parameterization of $w$ [110] while the right panel uses the same second order polynomial parameterization as Fig. 1. Phantom crossing was modeled using the prescription of Eq. (4.9). The constraints are best at $a \approx 0.8$ where we find that $w \approx-1 \pm 0.1$. At later times the constraints are weaker due to the integrated nature of the data. A very early times, $a \lesssim 0.4$, there is no strong lower bound on $w$ since very negative values make the dark energy just vanish more rapidly in the past.

For the constraints shown in Fig. 4 we use a likelihood that combines the Union 2 supernova compilation [128] and the WMAP 7-year data [129]. As models we choose two

\footnotetext{
${ }^{7}$ Available from http://cosmologist.info/cosmomc/

${ }^{8}$ Also available on David MacKay's website, http://www.inference.phy.cam.ac.uk/itprnn/book.html
} 
different parametrizations of the equation of state $w(a)$, the kink parameterization of [110] and a second-order polynomial form as used for Fig. 1 . We set $c_{s}^{2}=1$ and $\sigma=0$, which corresponds to a canonical scalar field model of dark energy (Quintessence). To these parameters we have to add the usual cosmological parameters $\left\{H_{0}, \Omega_{m}, \Omega_{b} h^{2}, A_{s}, n_{s}, \tau\right\}$ as well as possibly nuisance parameters required by the likelihood (none in our case since we marginalize analytically over the absolute supernova luminosity). Here the parameters $H_{0}$ and $\Omega_{m}$ describe together with $w(a)$ the background evolution (we take the universe to be spatially flat) while the baryon density $\Omega_{b}$, the reionisation optical depth $\tau$ as well as the amplitude of the primordial fluctuations $A_{s}$ and the scalar spectral index $n_{s}$ are necessary for the CMB predictions.

After performing the MCMC we end up with a chain consisting of a large number (in our case about $10^{5}$ ) of accepted parameter values. Each of these values encodes an evolution of $w(a)$. The figure then shows for each value of $a$ the location of the central $95 \%$ of $w(a)$ as the blue shaded region.

Instead of showing where most curves lie, we can also show the region in the $(w, a)$ plane where the dark energy evolution histories provide a "bad fit" to the data, relative to the best-fitting $w(a)$. Let us assume that at a given $a$ the probability distribution for $w(a)$ looks roughly like the Gaussian likelihood of Eq. (4.24), with $\mu$ being $w(a)$. The best fit (the maximum likelihood value) of (4.24) can easily be shown to lie at the arithmetic mean $\bar{x}$ with an uncertainty given by $\bar{\sigma}^{2}=\sigma^{2} / n$ (see e.g. chapter 24 of [123]), so that the distribution of the mean $\mu$ is again a normal distribution around $\bar{x}$ with variance $\bar{\sigma}^{2}$. The integral from $\bar{x}-2 \bar{\sigma}$ to $\bar{x}+2 \bar{\sigma}$ (the so-called two-sigma interval) encompasses about $95.4 \%$ of the probability for the location of $\mu$ (and the one sigma interval, i.e. the integral from $\bar{x}-\bar{\sigma}$ to $\bar{x}+\bar{\sigma}$, about $68.3 \%$ ). The quantity $\chi^{2}(\mu)=(\mu-\bar{x})^{2} / \bar{\sigma}^{2}$ increases by 4 when we move $\mu$ two sigma away from the best fit $\bar{x}$. We use this relationship and define an effective $\chi^{2}$ through $\chi_{\text {eff }}^{2}=-2 \ln \mathcal{L}$ which again for the simple case (4.24) agrees with $\chi^{2}$ up to an irrelevant normalization constant. We then plot in Fig. 4 also a red-shaded region showing the location of $w(a)$ for which $\chi_{\text {eff }}^{2}$ is larger by at least 4 than the smallest $\chi_{\text {eff }}^{2}$. This is then expected to delineate roughly the $2 \bar{\sigma}$ boundary for $w$ at a given $a$ and thus should approximately coincide with the boundary of the blue region, but shows the region where the badly fitting $w(a)$ lie.

We notice that the best constraints occur at $a \approx 0.8$ where we find $w \approx-1.0 \pm 0.1$ ( $1 \sigma$ errors). The constraints are compatible with $w=-1$ at all redshifts, the prediction for a cosmological constant. At higher redshift (smaller $a$ ) there is no lower bound for $w$ since the dark energy is subdominant in the past, and for very low values of $w$ it merely disappears faster as $a \rightarrow 0$. We also notice that while the red "exclusion" region is similar for the two parameterizations, the blue region for the kink model is narrower. This is an example of the prior imposed by a parameterization or model for $w$ on the results. This is not necessarily undesirable: the distance data is linked to $w$ through a double integration, so that the resulting constraints on the equation of state are strongly smoothed. If we would for example use higher and higher order polynomials then we would find less and less constraints as the resulting $w(a)$ would oscillate more and more around -1 . For this reason it is necessary to impose some constraint on the allowed form of $w$. For an example on how to do this in a Bayesian way with a maximum entropy prior, see [130].

\subsection{Modified Gravity models and anisotropic stresses}

So far we have been looking at models with fairly general pressure perturbations but still vanishing effective anisotropic stress. Is there a class of models for which it is natural to have 
$\sigma \neq 0$ in their effective energy momentum tensor, so that $\Pi=\phi-\psi \neq 0$ even when the contribution from relativistic particles is negligible? Indeed, there is: Let us look at a quite general scalar-tensor action, now including gravity and matter,

$$
S=\int d^{4} x \sqrt{-g}\left[\frac{1}{2}(1+f(\varphi)) R+K(X)-V(\varphi)+\mathcal{L}_{m}\right] .
$$

Here we have chosen a frame in which the matter is minimally coupled, so that it follows the geodesics of $g_{\mu \nu}$, since this is the frame what we would generally reconstruct from observations of weak lensing and the motion of galaxies [131]. We can again compute the Einstein equations by varying the action with respect to the metric, and arrange it in the Einstein form (3.2). In this case we find a total, effective dark energy EMT with $\Pi \propto f^{\prime}(\varphi)$ which does in general not vanish. This action therefore gives an explicit example of a model which has all the possible degrees of freedom that can be recovered from cosmological measurements ${ }^{9}$.

The scalar-tensor model is not the only example. Let us go through several typical "modified gravity" models (see e.g. [133] for a recent review). During this exercise we will also consider the question whether it is possible to revert to the case $\Pi=0$. For the scalartensor model it is easy to see that this requires $f(\varphi)$ to be constant. In other words, requiring the absence of anisotropic stress forces us to go to the GR limit of the theory. A class of models closely related to the scalar-tensor type is the is the $f(R)$ kind of models, with the gravitational part of the action given by

$$
S_{g} \sim \int d^{4} x \sqrt{-g} f(R)
$$

for an arbitrary function $f$. In this case, the effective anisotropic stress is found to be $\Pi \propto f^{\prime \prime}(R)$. A vanishing anisotropic stress then is only possible if $f(R)=R+\Lambda$ for a constant $\Lambda$, again just the GR action ${ }^{10}$. The problem can also be seen from a different angle by noticing that $f(R)$ models contain an effective scalar degree of freedom with a mass linked to the anisotropic stress (in the quasistatic limit) through $\Pi \propto 1 / m^{2}$. Turning off the anisotropic stress requires therefore to make this "scalaron" very massive and so effectively suppressing it, forcing the theory to revert to GR.

Another widely studied modified-gravity model is the so-called DGP (Dvali-GabadadzePorrati [134]) model which is based on a 4D brane embedded in a 5D bulk. The gravitational action is taken to be the 5D Einstein-Hilbert action together with an induced 4D EinsteinHilbert action confined to the brane. The relative strength of the two contributions is given by the crossover scale $r_{c}=M_{4}^{2} / M_{5}^{3}$. The effective anisotropic stress of the DGP model is proportional to $1 / \beta$ where $\beta=1+2 r_{c} H w_{\mathrm{DE}}$ (see (4.34) below). Setting the anisotropic stress to zero requires sending $\beta$ to infinity, which in turn only happens for $r_{c} \rightarrow \infty$, or $M_{5} \rightarrow 0$. But in this limit we have turned off the 5-dimensional nature of DGP gravity and are left with only the usual 4D Einstein-Hilbert action, and thus standard GR.

With two extra degrees of freedom on the other hand, it should be possible to balance them against each other and so turn off the anisotropic stress [135]. An example is afforded

\footnotetext{
${ }^{9}$ See also [132] where an action with a conformal coupling to the matter field is proposed as a generic way to parameterize modified-gravity models

${ }^{10}$ In principle the anisotropic stress also vanishes if $\delta R=0$ but this is a complicated condition on the evolution of the gravitational potentials that appears unnatural since it would require a very peculiar matter contribution to be compatible with the Einstein equations, and is at any rate not in agreement with observations.
} 
by the $f(R, G)$ type models [136] with action

$$
S_{g} \sim \int d^{4} x \sqrt{-g} f(R, G)
$$

where $G=R^{2}-4 R^{\mu \nu} R_{\mu \nu}+R^{\mu \nu \alpha \beta} R_{\mu \nu \alpha \beta}$ is the Gauss-Bonnet term, a topological invariant in $4 \mathrm{D}$ (i.e. it only contributes a boundary term in the action integral) ${ }^{11}$. Indeed it is now possible to build models that have no anisotropic stress, but in general the condition $\Pi=0$ depends on the background, i.e. a model has no anisotropic stress during e.g. matter domination but $\Pi \neq 0$ when accelerated expansion sets in. It should in principle be possible to create functions that both lead to the right sequence of evolutionary stages (radiation domination, then matter domination and finally accelerated expansion) and that retains $\Pi=0$, but it would be a quite complicated and fine-tuned endeavor, and it does not appear as if there was an easy way to link the anisotropic stress to the evolution, rather the contrary.

As an example, we fix the background evolution to be de Sitter. In that case we find that the condition $\Pi=0$ is equivalent to

$$
f_{, R R}+8 H_{0}^{2} f_{, R G}+16 H_{0}^{4} f_{, G G}=0 .
$$

The general solution of this equation is given by models of the form

$$
f(R, G)=f_{1}\left(R-\frac{G}{4 M^{2}}\right)+R f_{2}\left(R-\frac{G}{4 M^{2}}\right)
$$

for $M=H_{0}$ and $f_{1}, f_{2}$ two arbitrary functions. There are a few problems with this solution: Firstly one needs to end up in a de Sitter state with exactly the right expansion rate, given by the mass parameter in the original model, to have $\Pi=0$. And secondly, we found in [135] that the evolution of $H$ is unstable close to the de Sitter point for models of the type (4.30). In addition, also in these models, the mass of a scalaron degree of freedom has to diverge in order to force the anisotropic stress to vanish. This does not constitute a proof that it is impossible to have zero anisotropic stress for viable and non-trivial $f(R, G)$ models, but it is nonetheless surprising how difficult it is to set up such a scenario.

It may be instructive to also spend a few lines investigation the typical size of the anisotropic stress present in the DGP model. We choose this MG model since the $f(R)$ and scalar-tensor models can always be continuously extended to GR, and their free functional degree of freedom makes it difficult to consider 'the' typical model of their class. For DGP we only have a single free number to choose, the cross-over radius $r_{c}$. The background expansion (assuming flatness) in DGP is given by (e.g. [138])

$$
H^{2}-\frac{H}{r_{c}}=\frac{8 \pi G_{N}}{3} \rho_{m}
$$

We will consider the second term on the left, $H / r_{c}$, as providing the dark energy contribution to the expansion rate and so consider it as being effectively on the right hand side. If the Hubble parameter today is given by $H_{0}$ and the relative matter density today is taken to be $\Omega_{m}$, then we want for consistency $H_{0} / r_{c}=H_{0}^{2}\left(1-\Omega_{m}\right)$ or $r_{c}=1 /\left[H_{0}\left(1-\Omega_{m}\right)\right]$. As naively expected, we see that the crossover scale of DGP needs to be of the size of the horizon scale

\footnotetext{
${ }^{11}$ We use the rather complicated looking combination $G$ since the extra combinations of the curvature tensor will in general lead to higher order equations of motion. This in turn leads to problems because of the Ostrogradski instability [137]. This problem is avoided by using $R$ and $G$.
} 
today, which reveals the required fine-tuning in this model. Without going into a great deal more detail (see e.g. $[139,140]$ ), one finds for the perturbations that

$$
\begin{aligned}
k^{2} \phi & =-4 \pi G_{N} a^{2}\left(1-\frac{1}{3 \beta}\right) \rho_{m} \Delta_{m} \\
k^{2} \psi & =-4 \pi G_{N} a^{2}\left(1+\frac{1}{3 \beta}\right) \rho_{m} \Delta_{m}
\end{aligned}
$$

for $\beta \equiv 1-2 r_{c} H\left[1+\dot{H} /\left(3 H^{2}\right)\right]=1+2 r_{c} H w_{\mathrm{DE}}$, and the matter perturbation evolution proceeds as usual (i.e. as given by Eqs. (3.8) and (3.9) with $w=0, \delta p=0$ and $\sigma=0$ ). In terms of the relative parameterization of Eq. (3.16) we find therefore that

$$
\eta=\frac{2}{3 \beta-1} \text {. }
$$

Numerically, $\eta$ is small at high redshifts but tends towards $\eta \approx-0.44$ today for a flat Universe with $\Omega_{m}=0.3$. In other words, the gravitational slip $\Pi$ (or equivalently the effective anisotropic stress) in DGP is of a size comparable to the gravitational potentials themselves. We should note here that the extra factors of $1 \pm 1 /(3 \beta)$ in Eqs. (4.32) and (4.33) affect the growth of the perturbations as well, so that the value of the gravitational potentials is somewhat different than in a Quintessence model with the same equation of state parameter - but of course this is good since it implies that it is possible to measure the extra perturbation level parameters, at least if they are of the size found in DGP.

We have seen that it is very difficult to avoid generating a late-time effective anisotropic stress for the models that we would call "modified gravity models", and in addition the corresponding gravitational slip is generically very large, of the order of the gravitational potentials themselves. For this reason $\Pi$ is a key diagnostic in the phenomenological framework: If we find a deviation from $\Lambda \mathrm{CDM}$, then constraints on the anisotropic stress can help to identify a likely explanation: If $\Pi \neq 0$ then Quintessence and K-essence type models are ruled out and a modification of GR looks likely, while in the opposite case modified gravity models are disfavored and it appears more sensible to look for a minimally coupled field.

The ghost issues in DGP can be cured by adding more higher order terms (taking care to ensure that the equations of motion stay second order). The most general such theory was worked out in [141], but only recently have such theories become better known, like the Galileon [142] and similar models like Kinetic Gravity Braiding [93], massive gravity [143] and others more [144, 145], described in much more detail elsewhere in this volume [17].

Equations (4.32) and (4.33) have an interesting property: we see that the extra terms cancel for the lensing potential $\phi+\psi$. In other words, light is lensed by matter perturbations exactly as in GR, without any additional lensing. This offers another way to see why suppressing the anisotropic stress forces DGP to revert back to GR: if both $\phi-\psi$ and $\phi+\psi$ are just given by the usual GR expressions without any dark energy contribution, then the (effective) dark energy does not contain any fluctuations. But this in general only possible if the dark energy is a cosmological constant: due to the Bianchi identity and the conservation of the rest of the EMT, also the effective dark energy EMT is conserved. If $w \neq-1$ the conservation equations (3.8) and (3.9) couple the perturbations to the gravitational potentials, and with only one remaining function to choose $(\delta p)$ it is in general not possible to find a solution. So not only does $\sigma=0$ suppress all effective dark energy perturbations for such models, in general it is not even possible to achieve this self-consistently except for a 
$\Lambda$ CDM-like behavior. Therefore if we want to have a non-trivial modified-gravity model with $\phi=\psi$, then this model needs in general to change the lensing potential.

It is not only DGP that has this property where lensing is unaffected. On the other hand, the full Galileon case should change lensing [146]. I suspect that in this case it may be possible to construct an explicit example where the anisotropic stress vanishes without rendering the model unviable - but this still needs to be demonstrated. But also for Galileons I expect that in general the anisotropic stress is non-zero. The only way around the need to fine-tune $\sigma$ to vanish is to include the property already at the level of the action. I do not know what that condition translates into for a fully general model, but as mentioned above, for the action (4.26) the condition is that the coupling to $R$ vanishes [131]. At least for this class (and for the reason outlined above) "anisotropic stress" and "modification of gravity" are synonyms.

On the observational front, current data is consistent with no additional anisotropic stress (and indeed no detection of any dark energy perturbations), but with errors of order unity on the additional perturbation parameters $\eta$ (3.16) and $Q$ (3.15) or their equivalents. Future observations over the next one to two decades will provide much stronger constraints, reaching about a $10 \%$ accuracy on the perturbation parameters in several redshift and scale bins. For more details, see for example [71, 73, 75, 77, 78, 147-156]

\section{$5 \quad$ Limitations and challenges}

\subsection{The dark degeneracy}

Any dark components are only detectable through measurements of the left hand side of the Einstein equation (3.2), and the inferred properties on the energy momentum tensor on the right hand side. But on the right hand side there is only the total dark EMT. This means that a single dark component looks just the same as

$$
T_{\mu \nu}^{(\mathrm{dark})}=T_{\mu \nu}^{(\mathrm{DM})}+T_{\mu \nu}^{(\Lambda)}+T_{\mu \nu}^{(\mathrm{Q})}+T_{\mu \nu}^{(\mathrm{MG})}+\ldots
$$

An immediate consequence is that cosmological data will never be able to tell us that we have more than one general dark energy component. The only way to reach such a conclusion would as a probabilistic statement based on model predictions and Occams razor.

But the situation is actually worse: if we restrict ourselves to distance data (or more generally, data that constrains only the background evolution) and try to reconstruct directly an equation of state parameter that can fit the data in a model with dark matter and dark energy (a fairly common approach), we find that

$$
w(z)=\frac{H(z)^{2}-\frac{2}{3} H(z) H^{\prime}(z)(1+z)}{\Omega_{m} H_{0}^{2}(1+z)^{3}-H(z)^{2}} .
$$

Using a dark energy with this $w(z)$, we will always fit the (background) data which is here given as $H(z)$. But in this expression $\Omega_{m}$ is a free parameter that we can choose as we want [157]. We will therefore find a possible dark energy for any amount of dark matter. Indeed, we cannot even be sure that there is dark matter at all, maybe we are dealing with a single dark fluid. We can thus generate possible families of dark energy evolutions, parameterized by $\Omega_{m}$. Again, only theoretical prejudice or non-gravitational tests can break this degeneracy. It is for this reason that we plotted only the total $w$ in Fig. 1. This is all that we can learn from background data. 
The reason is that we tried to measure more degrees of freedom than are present in the energy momentum tensor. We can only measure one pressure, but instead we try to measure a general pressure and the amount of dark matter. Of course the degeneracy is broken when we put constraints on the dark energy pressure, e.g. if we demand a constant $w$. Then we can measure both $w$ and $\Omega_{m}$. But then again, the true dark energy may not be characterized by a constant $w$, so the extra information that we input into the analysis may be wrong. The existence of the degeneracy is also a good test for codes that try to reconstruct a fully general $w(z)$ : using only background data there should then be no constraints on $\Omega_{m}$.

What happens at the level of perturbations? There are two extra functions, $c_{s}^{2}$ and $\sigma$, that can be constrained. This is the reason why we could constrain in section 4.5 the equation of state $w(z)$ of Quintessence and simultaneously measure $\Omega_{m}$ : dark matter has $c_{s}^{2}=0$ and Quintessence $c_{s}^{2}=1$. If we tried to do the same with cold dark energy, characterized by a free $w(z)$ and $\left\{c_{s}^{2}=0, \sigma=0\right\}$ then we recreate the degeneracy between dark energy and dark matter, and in that case it is again impossible to measure $\Omega_{m}$, see Fig. 5 . The same is true if we leave $c_{s}^{2}$ and $\sigma$ free: In that case we are reconstructing the most general dark fluid (within first order perturbation theory and only considering scalar perturbations), and so any further freedom cannot be constrained $[158,159]$. This is just due to the way Einstein's equations work, and the phenomenological framework allows at least to diagnose the issue.
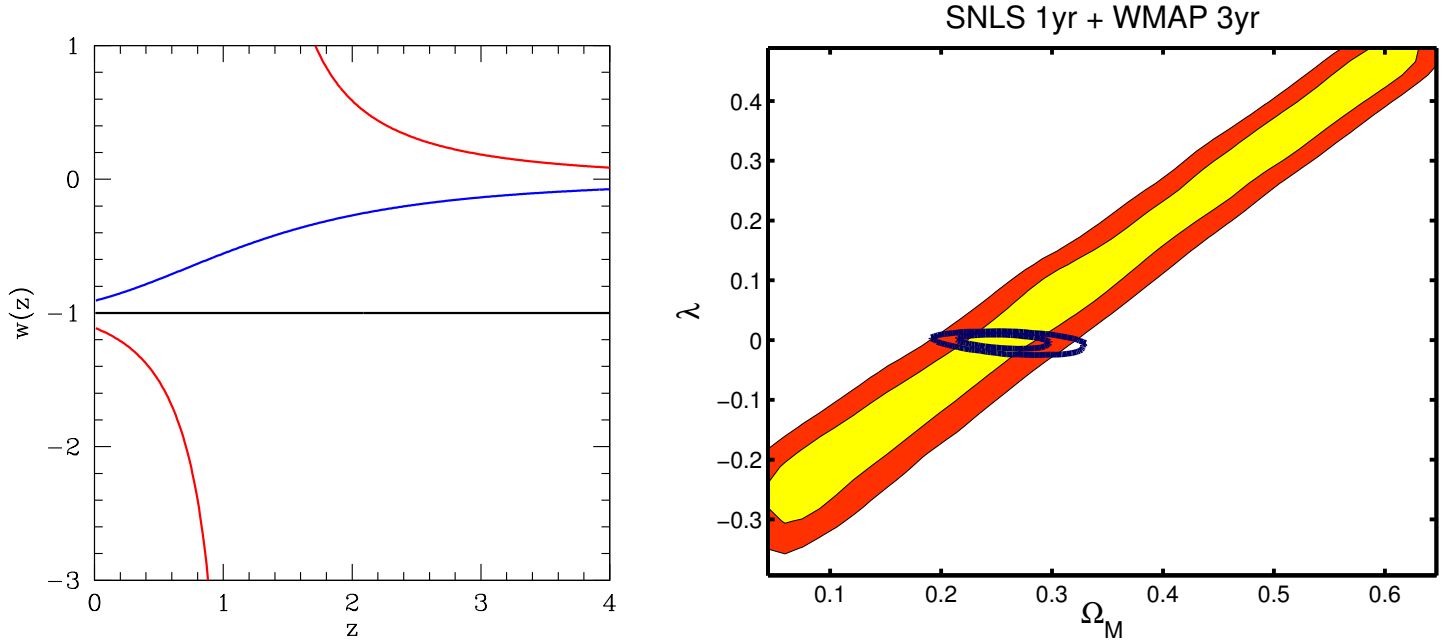

Figure 5. Left: Different $w_{\lambda}(z)=-1 /\left[1-\lambda(1+z)^{3}\right]$ with $\lambda=0$ (horizontal black line), $\lambda>0$ (red line starting at $w<-1$ ) and $\lambda<0$ (blue line starting at $w>-1$ ). All these $w$ 's lead to the same $\Lambda$ CDM expansion history if $\Omega_{m}$ is adjusted accordingly. Right: One and two sigma contours for a dark energy model given $w_{\lambda}(z), \sigma=0$ and $c_{s}^{2}=0$ (large yellow/red filled contours) and $c_{s}^{2}=1$ (black open contours). For $c_{s}^{2}=0$ we cannot distinguish between dark matter and dark energy and so cannot measure $\Omega_{m}$, while the choice $c_{s}^{2}=1$ breaks the degeneracy. This example taken from [157] uses the first-year SNLS supernova data [160] and the three-year WMAP data [161].

We also add more freedom when we introduce couplings between dark matter and dark energy. Again, since we can only measure the total dark energy momentum tensor, we can always separate it into an uncoupled sum of a dark matter and a general dark energy EMT, even if the the true model is actually a coupled model. We can always compute a "valid" 
dark energy EMT through

$$
T_{\mu \nu}^{(\mathrm{DE})}=T_{\mu \nu}^{(\text {total coupled })}-T_{\mu \nu}^{(\mathrm{DM})}
$$

for any choice of dark matter EMT. This means that we can always find an uncoupled dark energy - dark matter model that gives the same observations as a coupled model. Our only hope is that the coupled model is sufficiently well motivated on theoretical grounds as well as simpler to allow accepting it as the correct description. However, we can also do the inverse, we can always find a coupling function that will allow us to model the dark energy as a cosmological constant, as long as there is no additional anisotropic stress. To show this explicitly at the background level, let us assume that we have baryons and a coupled dark matter - dark energy fluid, and let us write the conservation equations for dark matter and dark energy as

$$
\begin{aligned}
\dot{\rho}_{\mathrm{DM}}+3 H \rho_{\mathrm{DM}} & =\rho_{\mathrm{DM}} C(t), \\
\dot{\rho}_{\mathrm{DE}}+3 H \rho_{\mathrm{DE}} & =-\rho_{\mathrm{DM}} C(t) .
\end{aligned}
$$

With this, and by assuming that the dark energy is a cosmological constant and computing $\dot{H}$ from the Friedmann equation, we find easily that [131]

$$
C(t)=\frac{\ddot{H}-\frac{9}{2} H \Omega_{b} H_{0}^{2} a^{-3}}{\dot{H}+\frac{3}{2} \Omega_{b} H_{0}^{2} a^{-3}}+3 H .
$$

This then is the (averaged) coupling that allows us to recover a cosmological constant as the dark energy. Again the problem is that the combination of coupling parameters and a general DE energy-momentum tensor encompasses more degrees of freedom that can be measured.

Part of the families of $w(a)$ that arise from the dark degeneracy, whether when changing $\Omega_{m}$ or couplings, contain phantom-crossing and even divergences at low redshift as can be seen in the left panel of Fig. 5. This typically happens if we need to "overcompensate" the dark energy. However, by construction the expansion rate $H(a)$ and therefore all observations are unaffected. This illustrates that strange behavior in $w$ and in other parameters (like the form of the effective sound speed of the Quintom model in figure 2), even divergences, are not necessarily problematic as long as the metric stays well behaved. Similar effects can apparently happen for $f(R)$ models [162]. Also coupled models can lead to (apparent) phantom crossing when they are analyzed without taking the coupling into account $[163,164]$; in this case this is directly the consequence of the dark degeneracy [157].

The dark degeneracy also shows that the dark energy does not need to be smooth at all. Current data is perfectly compatible with a clustering dark energy with $c_{s}^{2}=0$. It could contain some, or even all, of the clustering that is usually attributed to the dark matter. On the other hand, the current data is indeed compatible also with a smooth dark energy like a cosmological constant or a scalar field model with high sound speed. For this reason it is tempting to define the split between dark matter and dark energy by assigning the clustering part of the dark sector to the dark matter and the rest to the dark energy. This works for simple dark energy models like generalized quintessence since once we have defined the split in this way, the dark degeneracy guarantees that we can find a $w(z)$ for the dark energy that allows matching the observed $H(z)$. Although the split would not reflect physical reality if indeed the dark energy has a low sound speed and does cluster, we are barred by the dark degeneracy to see this with cosmological measurements alone. But if dark matter 
experiments one day indicate that the amount of dark matter is different from the amount observed in a cosmological setting, then we must not forget that the split of the dark sector into dark matter and dark energy is not unique, and that a different dark matter density can be accommodated by modifying the dark energy [159].

There is also a cosmological reason why it is a bad idea to use the clustering property directly to define the dark energy part of the total dark EMT and so break the dark degeneracy: modified gravity models tend to have $Q \neq 1$ in addition to $\eta \neq 0$. The nonzero anisotropic stress in these models contributes to the clustering through the last term in Eq. (3.9). By defining the dark energy to be smooth, we would exclude some of the models that we want to test with help of the data.

A closely related degeneracy exists between curvature and the dark energy $[165,166]$. Since the global curvature contributes only to the background expansion rate, it is easily confused with a smooth dark energy component. In order to break the curvature degeneracy, one needs to use a geometrical test for the presence of non-zero curvature, for example by comparing longitudinal and transverse distances [167]. So at least in principle it is possible to break the curvature degeneracy with cosmological observations (while the dark degeneracy is in principle perfect and unbreakable) but until this has been done one needs to be very careful not to mistake a small curvature contribution as a smooth, evolving dark energy.

\subsection{Non-linear evolution}

While the fundamental principle of measuring the geometry and then using Einstein's equations to map the results to an effective EMT (or other effective parameters) holds at the fully non-linear level, we have been working mostly with linear perturbation theory ${ }^{12}$. However, in the future we will want to consider at least mildly non-linear scales to make the best use of the data.

At the non-linear level we can expect some surprises to show up. Here just one example: when we look at the sky, we find that galaxies (and thus likely also the dark matter) are not static, but they move around. Such a motion induces a thermal pressure and anisotropic stress at second order even for pressureless dark matter [70],

$$
p_{m}=\frac{1}{3} v_{m}^{2} \rho_{m}, \quad \pi_{m}^{\mu \nu}=\rho_{m} v_{m}^{\langle\mu} v_{m}^{\nu\rangle} .
$$

(The combination $\pi_{m}^{\mu \nu}$ is the projected symmetric trace-free part, the fluid anisotropic stress $\sigma$ is related to its scalar part; see the appendix B.1 of [70] for the precise definitions.) This contribution is present even in $\Lambda \mathrm{CDM}$.

Since we can only observe the total dark EMT, we cannot distinguish the higher-order contributions to the dark matter pressure and anisotropic stress from the fundamental properties of the dark energy, another manifestation of the dark degeneracy of section 5.1. We can of course also not choose to measure only the linear perturbations, we always observe the fully non-linear result. We have to be very careful not to mix up such a non-linear contribution with the actual dark energy properties. Otherwise there is a risk that we will wrongly claim a detection of dark energy perturbations, ruling out $\Lambda$ CDM by mistake. However, at least on linear scales the peculiar velocity contribution to $\delta p$ and $\sigma$ is tiny and probably negligible [70].

\footnotetext{
${ }^{12}$ It is worth noting that the split of the energy-momentum tensor into an energy density, an isotropic pressure, an energy flux and an anisotropic stress can be defined at the fully non-linear level with the help of the covariant $1+3$ formalism, see e.g. [168].
} 


\subsection{Dependence on the environment}

A further issue relevant for the phenomenological description concerns a possible dependence on local physics. For example, a screening mechanism like the Vainshtein [169] or Chameleon [170-172] mechanism, based on local quantities like the density is an integral part of all viable MG models. Without such a screening these models all fall foul of local tests of gravity that place very strict limits on fifth forces in the solar system. But such a mechanism is difficult to model with the phenomenological description as it is not purely a function of scale $k$ [173], and a description at the level of the action that incorporates the screening effect may be preferable [132]. On the other hand, power spectra are also written as a function of scale and so phase the same problem. Environmental dependence is therefore a general concern for data analysis as a whole.

The precise way in which a given physical modified gravity model makes the transition from to the small-scale regime where the modifications are suppressed is actually an important discriminant: The action given in (4.26) already contains the possible degrees of freedom to first order in perturbation theory. Measuring the evolution of the Universe on large scales will allow to constrain the functions $f(\varphi)$ (through the anisotropic stress), $V(\varphi)$ (mostly through the background expansion) and $K(X)$ (mostly through the sound speed). But this is certainly not the only action with these degrees of freedom that can be written down, and there is a degeneracy between different actions that can at least partially be broken by considering the additional information from the way the model reverts to GR on small scales.

\section{Conclusions and Outlook}

In this review we have discussed a general framework that allows to parameterize all possible outcomes of cosmological observations. ${ }^{13}$ We found that this can be done in different ways that are all directly related but may be better in some situation or other. The first approach is to use an effective dark energy momentum tensor, where the relevant degrees of freedom can be taken to be the average (background) pressure $p(t)$, and the pressure perturbation $\delta p(t, k)$ as well as the anisotropic stress $\sigma(t, k)$. Usually the background pressure is given in terms of an equation of state parameter $w=p / \rho$, and often the pressure perturbation is parameterized by a rest-frame sound speed $c_{s}^{2}$.

The Einstein equations relate these quantities to the geometry, where the equivalent degrees of freedom are the average expansion rate $H$ and (in the conformal Newtonian gauge) the gravitational potentials $\phi$ and $\psi$. There are many ways to introduce other parameterizations, a common approach is to use $w$ and in addition parameters that quantify the deviation from a hypothetical universe where the gravitational potentials are given by the perturbations in the matter (and radiation) alone. The main point here is that one always needs one background parameter and two perturbation level parameters for all measurements that involve perturbations, like the CMB, galaxy clustering, weak lensing, redshift space distortions, etc. Conversely, these $1+2$ functions also represent the information that can be extracted from measurements.

In this way we have built a framework that allows to combine all possible cosmological observations, by parameterizing exactly the possible degrees of freedom. With this, we are

\footnotetext{
${ }^{13}$ Small print: At least to first order and for scalar perturbations. The latter is straightforward to rectify by including vector and tensor perturbations, while extensions to non-linear perturbations present a more formidable challenge, at least concerning the interpretation of the results.
} 
also sure that we can probe the consistency of observations with the still favored $\Lambda$ CDM model in all possible ways.

We have further seen that the phenomenological parameters can be mapped to functions in an action that describes gravity and the fields present in the Universe. Through this we can reconstruct actions from the observations, but again the limited information available will mean that there are degeneracies that cannot be broken. Nonetheless the phenomenological parameters will give an indication of which classes of models we should investigate in more detail. As an example, the presence of a strong late-time anisotropic stress appears closely related to a modification of gravity.

Nonetheless, the framework rests on the validity of the cosmological principle, i.e. statistical isotropy and homogeneity. If the Universe has a fundamentally different structure, e.g. if we lived in the center of a gigantic void, then further degrees of freedom become possible. As an example, in such a case there are two different functions that parameterize the background evolution, that can be taken to be a transverse and a longitudinal expansion rate. In a FLRW background they are equal, but in a Lemaitre-Tolman-Bondi (LTB) background they are in general different. It has been shown that in this case they can be distinguished by measurements of $H(z)$ and distances $[167,174]$. The full perturbation theory for the LTB background is still being worked out, and there are of course further generalizations possible. Hence when using the phenomenological approach one should always be aware of the FLRW assumption that has been made, and one should test this assumption as far as possible.

Today the phenomenological parameters are not well constrained, with deviations of order unity still possible. However, future very large surveys like EuCLID [1] will significantly tighten the bounds and will permit to constrain $w$ at the $1 \%$ level and the perturbative quantities at the $10 \%$ level $[71,73,75,77,78,147-156]$. To reach this precision will still take a lot of work to control the systematic errors in the observations, but also to ensure that all important theoretical effects are taken into account, for example non-linear behavior of the perturbations, but also relativistic effects [175-178] as well as including vector and tensor modes.

\section{Acknowledgments}

I would like to thank Philippe Brax and Céline Boehm for inviting me to write this minireview. It is a pleasure to thank all my dark energy collaborators over the years, and I am especially grateful to Bruce Bassett, Philippe Brax, Ruth Durrer, Lukas Hollenstein, Dragan Huterer and Ignacy Sawicki for helpful discussions and comments about this manuscript. This work is supported by the Swiss National Science Foundation. I gratefully acknowledge hospitality by UNFPA Thailand during part of the writing of this review.

\section{References}

[1] Euclid Consortium Collaboration, R. Laureijs, J. Amiaux, S. Arduini, J.-L. Augueres, et. al., Euclid Definition Study Report, arXiv:1110.3193.

[2] A. Einstein, The Field Equations of Gravitation, Sitzungsber.Preuss.Akad.Wiss.Berlin (Math.Phys.) 1915 (1915) 844-847.

[3] A. Einstein, Cosmological Considerations in the General Theory of Relativity, Sitzungsber.Preuss.Akad.Wiss.Berlin (Math.Phys.) 1917 (1917) 142-152. 
[4] Supernova Search Team Collaboration, A. G. Riess et. al., Observational evidence from supernovae for an accelerating universe and a cosmological constant, Astron.J. 116 (1998) 1009-1038, [astro-ph/9805201].

[5] Supernova Cosmology Project Collaboration, S. Perlmutter et. al., Measurements of Omega and Lambda from 42 high redshift supernovae, Astrophys.J. 517 (1999) 565-586, [astro-ph/9812133].

[6] S. M. Carroll, The Cosmological constant, Living Rev.Rel. 4 (2001) 1, [astro-ph/0004075].

[7] E. J. Copeland, M. Sami, and S. Tsujikawa, Dynamics of dark energy, Int.J.Mod.Phys. D15 (2006) 1753-1936, [hep-th/0603057].

[8] R. Durrer and R. Maartens, Dark Energy and Modified Gravity, arXiv:0811.4132.

[9] P. Brax, Gif Lectures on Cosmic Acceleration, arXiv:0912.3610.

[10] B. Jain and J. Khoury, Cosmological Tests of Gravity, Annals Phys. 325 (2010) 1479-1516, [arXiv: 1004.3294].

[11] D. Sapone, Dark Energy in Practice, Int.J.Mod.Phys. A25 (2010) 5253-5331, [arXiv:1006.5694].

[12] T. Clifton, P. G. Ferreira, A. Padilla, and C. Skordis, Modified Gravity and Cosmology, arXiv:1106.2476.

[13] L. Amendola and S. Tsujikawa, Dark Energy: Theory and Observations. 2010.

[14] P. Astier and R. Pain, Observational Evidence of the Accelerated Expansion of the Universe, arXiv: 1204. xxxx.

[15] J. Martin, Everything You always Wanted to Know about the Cosmological Constant (but Were Afraid to Ask), arXiv:1204.xxxx.

[16] C. Clarkson, Establishing Homogeneity of the Universe in the Shadow of Dark Energy, arXiv:1204.xxxx.

[17] C. de Rham, Galileons in the Sky, arXiv:1204.xxxx.

[18] I. M. H. Etherington, On the Definition of Distance in General Relativity., Philosophical Magazine 15 (1933) 761.

[19] B. A. Bassett and M. Kunz, Cosmic distance-duality as a probe of exotic physics and acceleration, Phys.Rev. D69 (2004) 101305, [astro-ph/0312443].

[20] B. A. Bassett and M. Kunz, Cosmic Acceleration versus Axion-Photon Mixing, Astrophys. J. 607 (June, 2004) 661-664, [astro-ph/0311495].

[21] J.-P. Uzan, N. Aghanim, and Y. Mellier, The Distance duality relation from x-ray and SZ observations of clusters, Phys.Rev. D70 (2004) 083533, [astro-ph/0405620].

[22] A. Avgoustidis, C. Burrage, J. Redondo, L. Verde, and R. Jimenez, Constraints on cosmic opacity and beyond the standard model physics from cosmological distance measurements, JCAP 1010 (2010) 024, [arXiv:1004.2053].

[23] M. Kunz and B. A. Bassett, A tale of two distances, astro-ph/0406013.

[24] C. Clarkson, G. Ellis, A. Faltenbacher, R. Maartens, O. Umeh, et. al., (Mis-)Interpreting supernovae observations in a lumpy universe, arXiv:1109.2484.

[25] M. Maggiore, L. Hollenstein, M. Jaccard, and E. Mitsou, Early dark energy from zero-point quantum fluctuations, Phys.Lett. B704 (2011) 102-107, [arXiv:1104.3797].

[26] L. Hollenstein, M. Jaccard, M. Maggiore, and E. Mitsou, Zero-point quantum fluctuations in cosmology, arXiv:1111.5575.

[27] A. R. Liddle and D. Lyth, Cosmological inflation and large scale structure, . 
[28] WMAP Collaboration Collaboration, E. Komatsu et. al., Seven-Year Wilkinson Microwave Anisotropy Probe (WMAP) Observations: Cosmological Interpretation, Astrophys.J.Suppl. 192 (2011) 18, [arXiv:1001.4538].

[29] S. Ilic, M. Kunz, A. R. Liddle, and J. A. Frieman, A dark energy view of inflation, Phys.Rev. D81 (2010) 103502, [arXiv:1002.4196].

[30] T. Buchert, On average properties of inhomogeneous fluids in general relativity. 1. Dust cosmologies, Gen.Rel.Grav. 32 (2000) 105-125, [gr-qc/9906015].

[31] S. Rasanen, Dark energy from backreaction, JCAP 0402 (2004) 003, [astro-ph/0311257].

[32] S. Rasanen, Accelerated expansion from structure formation, JCAP 0611 (2006) 003, [astro-ph/0607626].

[33] T. Buchert, Dark Energy from Structure: A Status Report, Gen.Rel.Grav. 40 (2008) 467-527, [arXiv: 0707.2153].

[34] J. Moffat and D. Tatarski, Redshift and structure formation in a spatially flat inhomogeneous universe, Phys.Rev. D45 (1992) 3512-3522.

[35] K. Tomita, A local void and the accelerating universe, Mon.Not.Roy.Astron.Soc. 326 (2001) 287, [astro-ph/0011484].

[36] M.-N. Celerier, Do we really see a cosmological constant in the supernovae data?, Astron.Astrophys. 353 (2000) 63-71, [astro-ph/9907206].

[37] T. Biswas, R. Mansouri, and A. Notari, Nonlinear Structure Formation and Apparent Acceleration: An Investigation, JCAP 0712 (2007) 017, [astro-ph/0606703].

[38] J. Garcia-Bellido and T. Haugboelle, Confronting Lemaitre-Tolman-Bondi models with Observational Cosmology, JCAP 0804 (2008) 003, [arXiv: 0802.1523].

[39] C. Clarkson and R. Maartens, Inhomogeneity and the foundations of concordance cosmology, Class.Quant.Grav. 27 (2010) 124008, [arXiv:1005.2165]. 26 pages and 1 figure. Invited review article for the CQG special issue on nonlinear cosmological perturbations. v2 has additional refs and comments, minor errors corrected, version in CQG.

[40] J. Larena, J.-M. Alimi, T. Buchert, M. Kunz, and P.-S. Corasaniti, Testing backreaction effects with observations, Phys.Rev. D79 (2009) 083011, [arXiv:0808.1161].

[41] A. D. Linde, D. A. Linde, and A. Mezhlumian, Do we live in the center of the world?, Phys.Lett. B345 (1995) 203-210, [hep-th/9411111].

[42] D. Parkinson, B. A. Bassett, and J. D. Barrow, Mapping the dark energy with varying alpha, Phys.Lett. B578 (2004) 235-240, [astro-ph/0307227].

[43] J.-P. Uzan, Varying Constants, Gravitation and Cosmology, Living Rev.Rel. 14 (2011) 2, [arXiv: 1009.5514].

[44] D. Huterer and M. S. Turner, Probing the dark energy: Methods and strategies, Phys.Rev. D64 (2001) 123527, [astro-ph/0012510].

[45] I. Maor, R. Brustein, and P. J. Steinhardt, Limitations in using luminosity distance to determine the equation of state of the universe, Phys.Rev.Lett. 86 (2001) 6, [astro-ph/0007297].

[46] J. Weller and A. Albrecht, Opportunities for future supernova studies of cosmic acceleration, Phys.Rev.Lett. 86 (2001) 1939-1942, [astro-ph/0008314].

[47] M. Chevallier and D. Polarski, Accelerating universes with scaling dark matter, Int.J.Mod.Phys. D10 (2001) 213-224, [gr-qc/0009008].

[48] E. V. Linder, Exploring the expansion history of the universe, Phys.Rev.Lett. 90 (2003) 091301, [astro-ph/0208512]. 
[49] P. S. Corasaniti and E. Copeland, A Model independent approach to the dark energy equation of state, Phys.Rev. D67 (2003) 063521, [astro-ph/0205544].

[50] M. Douspis, Y. Zolnierowski, A. Blanchard, and A. Riazuelo, What can we learn about dark energy evolution?, Astron.Astrophys. (2006) [astro-ph/0602491].

[51] B. A. Bassett, M. Kunz, J. Silk, and C. Ungarelli, A Late time transition in the cosmic dark energy?, Mon.Not.Roy.Astron.Soc. 336 (2002) 1217-1222, [astro-ph/0203383].

[52] B. A. Bassett, P. S. Corasaniti, and M. Kunz, The Essence of quintessence and the cost of compression, Astrophys.J. 617 (2004) L1-L4, [astro-ph/0407364].

[53] D. Huterer and G. Starkman, Parameterization of dark-energy properties: A Principal-component approach, Phys.Rev.Lett. 90 (2003) 031301, [astro-ph/0207517].

[54] T. Holsclaw, U. Alam, B. Sanso, H. Lee, K. Heitmann, et. al., Nonparametric Dark Energy Reconstruction from Supernova Data, Phys.Rev.Lett. 105 (2010) 241302, [arXiv:1011.3079].

[55] Y. Wang and P. M. Garnavich, Measuring time dependence of dark energy density from type Ia supernova data, Astrophys.J. 552 (2001) 445, [astro-ph/0101040].

[56] M. Tegmark, Measuring the metric: A Parametrized postFriedmanian approach to the cosmic dark energy problem, Phys.Rev. D66 (2002) 103507, [astro-ph/0101354].

[57] R. A. Daly and S. Djorgovski, A model-independent determination of the expansion and acceleration rates of the universe as a function of redshift and constraints on dark energy, Astrophys.J. 597 (2003) 9-20, [astro-ph/0305197].

[58] M. Kunz, A. R. Liddle, D. Parkinson, and C. Gao, Constraining the dark fluid, Phys.Rev. D80 (2009) 083533, [arXiv:0908.3197].

[59] Supernova Cosmology Project Collaboration, M. Kowalski et. al., Improved Cosmological Constraints from New, Old and Combined Supernova Datasets, Astrophys.J. 686 (2008) 749-778, [arXiv:0804.4142].

[60] Y. Wang and P. Mukherjee, Observational Constraints on Dark Energy and Cosmic Curvature, Phys.Rev. D76 (2007) 103533, [astro-ph/0703780].

[61] SDSS Collaboration Collaboration, B. A. Reid et. al., Baryon Acoustic Oscillations in the Sloan Digital Sky Survey Data Release 7 Galaxy Sample, Mon.Not.Roy.Astron.Soc. 401 (2010) 2148-2168, [arXiv:0907.1660].

[62] A. G. Riess, L. Macri, S. Casertano, M. Sosey, H. Lampeitl, et. al., A Redetermination of the Hubble Constant with the Hubble Space Telescope from a Differential Distance Ladder, Astrophys.J. 699 (2009) 539-563, [arXiv:0905.0695].

[63] H. Kodama and M. Sasaki, Cosmological Perturbation Theory, Prog.Theor.Phys.Suppl. 78 (1984) 1-166.

[64] R. Durrer, Gauge invariant cosmological perturbation theory: A General study and its application to the texture scenario of structure formation, Fund.Cosmic Phys. 15 (1994) 209, [astro-ph/9311041].

[65] C.-P. Ma and E. Bertschinger, Cosmological perturbation theory in the synchronous and conformal Newtonian gauges, Astrophys.J. 455 (1995) 7-25, [astro-ph/9506072].

[66] W. Hu, Covariant linear perturbation formalism, astro-ph/0402060.

[67] K. A. Malik and D. Wands, Cosmological perturbations, Phys.Rept. 475 (2009) 1-51, [arXiv:0809.4944].

[68] H. Sandvik, M. Tegmark, M. Zaldarriaga, and I. Waga, The end of unified dark matter?, Phys.Rev. D69 (2004) 123524, [astro-ph/0212114].

[69] R. Bean and O. Dore, Are Chaplygin gases serious contenders to the dark energy throne?, 
Phys.Rev. D68 (2003) 023515, [astro-ph/0301308].

[70] G. Ballesteros, L. Hollenstein, R. K. Jain, and M. Kunz, Nonlinear cosmological consistency relations and effective matter stresses, arXiv:1112.4837.

[71] L. Amendola, M. Kunz, and D. Sapone, Measuring the dark side (with weak lensing), JCAP 0804 (2008) 013, [arXiv:0704.2421].

[72] S. F. Daniel, E. V. Linder, T. L. Smith, R. R. Caldwell, A. Cooray, et. al., Testing General Relativity with Current Cosmological Data, Phys.Rev. D81 (2010) 123508, [arXiv: 1002.1962].

[73] R. Bean and M. Tangmatitham, Current constraints on the cosmic growth history, Phys.Rev. D81 (2010) 083534, [arXiv:1002.4197].

[74] P. G. Ferreira and C. Skordis, The linear growth rate of structure in Parametrized Post Friedmannian Universes, Phys.Rev. D81 (2010) 104020, [arXiv:1003.4231].

[75] L. Pogosian, A. Silvestri, K. Koyama, and G.-B. Zhao, How to optimally parametrize deviations from General Relativity in the evolution of cosmological perturbations?, Phys.Rev. D81 (2010) 104023, [arXiv:1002.2382].

[76] G.-B. Zhao, T. Giannantonio, L. Pogosian, A. Silvestri, D. J. Bacon, et. al., Probing modifications of General Relativity using current cosmological observations, Phys.Rev. D81 (2010) 103510, [arXiv:1003.0001].

[77] S. F. Daniel and E. V. Linder, Confronting General Relativity with Further Cosmological Data, Phys.Rev. D82 (2010) 103523, [arXiv:1008.0397].

[78] W. Hu and I. Sawicki, A Parameterized Post-Friedmann Framework for Modified Gravity, Phys.Rev. D76 (2007) 104043, [arXiv: 0708.1190].

[79] W. Hu, Structure formation with generalized dark matter, Astrophys.J. 506 (1998) 485-494, [astro-ph/9801234].

[80] R. Caldwell, R. Dave, and P. J. Steinhardt, Cosmological imprint of an energy component with general equation of state, Phys.Rev.Lett. 80 (1998) 1582-1585, [astro-ph/9708069].

[81] C. Wetterich, Cosmology and the Fate of Dilatation Symmetry, Nucl.Phys. B302 (1988) 668.

[82] B. Ratra and P. Peebles, Cosmological Consequences of a Rolling Homogeneous Scalar Field, Phys.Rev. D37 (1988) 3406.

[83] M. Kunz and D. Sapone, Crossing the Phantom Divide, Phys.Rev. D74 (2006) 123503, [astro-ph/0609040].

[84] D. Huterer and M. S. Turner, Prospects for probing the dark energy via supernova distance measurements, Phys.Rev. D60 (1999) 081301, [astro-ph/9808133].

[85] T. D. Saini, S. Raychaudhury, V. Sahni, and A. A. Starobinsky, Reconstructing the cosmic equation of state from supernova distances, Phys.Rev.Lett. 85 (2000) 1162-1165, [astro-ph/9910231].

[86] M. Sahlen, A. R. Liddle, and D. Parkinson, Direct reconstruction of the quintessence potential, Phys.Rev. D72 (2005) 083511, [astro-ph/0506696].

[87] T. Chiba, T. Okabe, and M. Yamaguchi, Kinetically driven quintessence, Phys.Rev. D62 (2000) 023511, [astro-ph/9912463].

[88] C. Armendariz-Picon, V. F. Mukhanov, and P. J. Steinhardt, A Dynamical solution to the problem of a small cosmological constant and late time cosmic acceleration, Phys.Rev.Lett. $\mathbf{8 5}$ (2000) 4438-4441, [astro-ph/0004134].

[89] C. Bonvin, C. Caprini, and R. Durrer, A no-go theorem for $k$-essence dark energy, Phys.Rev.Lett. 97 (2006) 081303, [astro-ph/0606584]. 
[90] C. Bonvin, C. Caprini, and R. Durrer, Superluminal motion and closed signal curves, arXiv:0706.1538.

[91] E. Babichev, V. Mukhanov, and A. Vikman, k-Essence, superluminal propagation, causality and emergent geometry, JHEP 0802 (2008) 101, [arXiv:0708.0561].

[92] S. M. Carroll, M. Hoffman, and M. Trodden, Can the dark energy equation of state parameter $w$ be less than -1?, Phys.Rev. D68 (2003) 023509, [astro-ph/0301273].

[93] C. Deffayet, O. Pujolas, I. Sawicki, and A. Vikman, Imperfect Dark Energy from Kinetic Gravity Braiding, JCAP 1010 (2010) 026, [arXiv: 1008.0048].

[94] P. Creminelli, G. D'Amico, J. Norena, and F. Vernizzi, The Effective Theory of Quintessence: the $w<-1$ Side Unveiled, JCAP 0902 (2009) 018, [arXiv:0811.0827].

[95] E. A. Lim, I. Sawicki, and A. Vikman, Dust of Dark Energy, JCAP 1005 (2010) 012, [arXiv: 1003.5751].

[96] B. Feng, X.-L. Wang, and X.-M. Zhang, Dark energy constraints from the cosmic age and supernova, Phys.Lett. B607 (2005) 35-41, [astro-ph/0404224].

[97] W. Hu, Crossing the phantom divide: Dark energy internal degrees of freedom, Phys.Rev. D71 (2005) 047301, [astro-ph/0410680].

[98] D. Sapone, M. Kunz, and M. Kunz, Fingerprinting Dark Energy, Phys.Rev. D80 (2009) 083519, [arXiv: 0909.0007].

[99] D. Sapone, M. Kunz, and L. Amendola, Fingerprinting Dark Energy II: weak lensing and galaxy clustering tests, Phys.Rev. D82 (2010) 103535, [arXiv:1007.2188].

[100] R. de Putter, D. Huterer, and E. V. Linder, Measuring the Speed of Dark: Detecting Dark Energy Perturbations, Phys.Rev. D81 (2010) 103513, [arXiv:1002.1311].

[101] G. Ballesteros and J. Lesgourgues, Dark energy with non-adiabatic sound speed: initial conditions and detectability, JCAP 1010 (2010) 014, [arXiv:1004.5509].

[102] S. Perlmutter, M. S. Turner, and M. J. White, Constraining dark energy with SNe Ia and large scale structure, Phys.Rev.Lett. 83 (1999) 670-673, [astro-ph/9901052].

[103] P. S. Corasaniti and E. J. Copeland, Constraining the quintessence equation of state with SnIa data and CMB peaks, Phys.Rev. D65 (2002) 043004, [astro-ph/0107378].

[104] J. Weller and A. Lewis, Large scale cosmic microwave background anisotropies and dark energy, Mon.Not.Roy.Astron.Soc. 346 (2003) 987-993, [astro-ph/0307104].

[105] C. Baccigalupi, A. Balbi, S. Matarrese, F. Perrotta, and N. Vittorio, Constraints on flat cosmologies with tracking quintessence from cosmic microwave background observations, Phys.Rev. D65 (2002) 063520, [astro-ph/0109097].

[106] R. Bean and A. Melchiorri, Current constraints on the dark energy equation of state, Phys.Rev. D65 (2002) 041302, [astro-ph/0110472].

[107] B. A. Bassett, M. Kunz, D. Parkinson, and C. Ungarelli, Condensate cosmology - Dark energy from dark matter, Phys.Rev. D68 (2003) 043504, [astro-ph/0211303].

[108] R. Bean and O. Dore, Probing dark energy perturbations: The Dark energy equation of state and speed of sound as measured by WMAP, Phys.Rev. D69 (2004) 083503, [astro-ph/0307100].

[109] M. Kunz, P.-S. Corasaniti, D. Parkinson, and E. J. Copeland, Model-independent dark energy test with sigma(8) using results from the Wilkinson microwave anisotropy probe, Phys.Rev. D70 (2004) 041301, [astro-ph/0307346]. Reviewed in Nature 431:519,2004.

[110] P. S. Corasaniti, M. Kunz, D. Parkinson, E. Copeland, and B. Bassett, The Foundations of observing dark energy dynamics with the Wilkinson Microwave Anisotropy Probe, Phys.Rev. 
D70 (2004) 083006, [astro-ph/0406608].

[111] U. Seljak and M. Zaldarriaga, A Line of sight integration approach to cosmic microwave background anisotropies, Astrophys.J. 469 (1996) 437-444, [astro-ph/9603033].

[112] A. Lewis, A. Challinor, and A. Lasenby, Efficient computation of CMB anisotropies in closed FRW models, Astrophys.J. 538 (2000) 473-476, [astro-ph/9911177].

[113] A. R. Liddle, How many cosmological parameters?, Mon.Not.Roy.Astron.Soc. 351 (2004) L49-L53, [astro-ph/0401198].

[114] T. D. Saini, J. Weller, and S. Bridle, Revealing the nature of dark energy using Bayesian evidence, Mon.Not.Roy.Astron.Soc. 348 (2004) 603, [astro-ph/0305526].

[115] R. Trotta, Applications of Bayesian model selection to cosmological parameters, Mon.Not.Roy.Astron.Soc. 378 (2007) 72-82, [astro-ph/0504022].

[116] M. Kunz, R. Trotta, and D. Parkinson, Measuring the effective complexity of cosmological models, Phys.Rev. D74 (2006) 023503, [astro-ph/0602378].

[117] E. T. Jaynes and G. L. Bretthorst, Probability Theory. Cambridge University Press, June, 2003.

[118] W. Feller, An introduction to probability theory and its applications. Vol. I. Third edition. John Wiley \& Sons Inc., New York, 1968.

[119] W. Feller, An introduction to probability theory and its applications. Vol. II. Second edition. John Wiley \& Sons Inc., New York, 1971.

[120] N. Metropolis, A. Rosenbluth, M. Rosenbluth, A. Teller, and E. Teller, Equation of state calculations by fast computing machines, J.Chem.Phys. 21 (1953) 1087-1092.

[121] A. Lewis and S. Bridle, Cosmological parameters from CMB and other data: A Monte Carlo approach, Phys.Rev. D66 (2002) 103511, [astro-ph/0205436].

[122] A. Lewis and S. Bridle, "CosmoMC Notes." http://cosmologist.info/notes/CosmoMC.pdf.

[123] D. MacKay, Information theory, inference, and learning algorithms. Cambridge University Press, 2003.

[124] J. Skilling, Nested Sampling, in American Institute of Physics Conference Series (R. Fischer, R. Preuss, and U. V. Toussaint, eds.), pp. 395-405, Nov., 2004.

[125] P. Mukherjee, D. Parkinson, and A. R. Liddle, A nested sampling algorithm for cosmological model selection, Astrophys.J. 638 (2006) L51-L54, [astro-ph/0508461].

[126] F. Feroz and M. Hobson, Multimodal nested sampling: an efficient and robust alternative to MCMC methods for astronomical data analysis, Mon.Not.Roy.Astron.Soc. 384 (2008) 449, [arXiv: 0704.3704].

[127] D. Wraith, M. Kilbinger, K. Benabed, O. Cappe, J.-F. Cardoso, et. al., Estimation of cosmological parameters using adaptive importance sampling, Phys.Rev. D80 (2009) 023507, [arXiv: 0903.0837].

[128] R. Amanullah, C. Lidman, D. Rubin, G. Aldering, P. Astier, et. al., Spectra and Light Curves of Six Type Ia Supernovae at $0.511<z<1.12$ and the Union2 Compilation, Astrophys.J. 716 (2010) 712-738, [arXiv: 1004.1711].

[129] D. Larson, J. Dunkley, G. Hinshaw, E. Komatsu, M. Nolta, et. al., Seven-Year Wilkinson Microwave Anisotropy Probe (WMAP) Observations: Power Spectra and WMAP-Derived Parameters, Astrophys.J.Suppl. 192 (2011) 16, [arXiv:1001.4635].

[130] C. Zunckel and R. Trotta, Reconstructing the history of dark energy using maximum entropy, Mon.Not.Roy.Astron.Soc. 380 (2007) 865, [astro-ph/0702695]. 
[131] L. Amendola, M. Kunz, I. Saltas, and I. Sawicki, All you can know about Dark Energy, . in preparation.

[132] P. Brax, A.-C. Davis, and B. Li, Modified Gravity Tomography, arXiv:1111.6613.

[133] S. Tsujikawa, Modified gravity models of dark energy, Lect.Notes Phys. 800 (2010) 99-145, [arXiv:1101.0191].

[134] G. Dvali, G. Gabadadze, and M. Porrati, 4-D gravity on a brane in 5-D Minkowski space, Phys.Lett. B485 (2000) 208-214, [hep-th/0005016].

[135] I. D. Saltas and M. Kunz, Anisotropic stress and stability in modified gravity models, Phys.Rev. D83 (2011) 064042, [arXiv:1012.3171].

[136] A. De Felice and T. Suyama, Vacuum structure for scalar cosmological perturbations in Modified Gravity Models, JCAP 0906 (2009) 034, [arXiv:0904.2092].

[137] R. P. Woodard, Avoiding dark energy with 1/r modifications of gravity, Lect.Notes Phys. 720 (2007) 403-433, [astro-ph/0601672].

[138] R. Maartens and E. Majerotto, Observational constraints on self-accelerating cosmology, Phys.Rev. D74 (2006) 023004, [astro-ph/0603353].

[139] A. Lue, R. Scoccimarro, and G. D. Starkman, Probing Newton's constant on vast scales: DGP gravity, cosmic acceleration and large scale structure, Phys.Rev. D69 (2004) 124015, [astro-ph/0401515].

[140] K. Koyama and R. Maartens, Structure formation in the dgp cosmological model, JCAP 0601 (2006) 016, [astro-ph/0511634].

[141] G. W. Horndeski, Second-order scalar-tensor field equations in a four-dimensional space, International Journal of Theoretical Physics 10 (1974) 363-384. 10.1007/BF01807638.

[142] A. Nicolis, R. Rattazzi, and E. Trincherini, The Galileon as a local modification of gravity, Phys.Rev. D79 (2009) 064036, [arXiv:0811.2197].

[143] C. de Rham and G. Gabadadze, Generalization of the Fierz-Pauli Action, Phys.Rev. D82 (2010) 044020, [arXiv:1007.0443].

[144] C. Charmousis, E. J. Copeland, A. Padilla, and P. M. Saffin, General second order scalar-tensor theory, self tuning, and the Fab Four, arXiv:1106.2000.

[145] A. De Felice and S. Tsujikawa, Conditions for the cosmological viability of the most general scalar-tensor theories and their applications to extended Galileon dark energy models, arXiv: 1110.3878.

[146] M. Wyman, Galilean-invariant scalar fields can strengthen gravitational lensing, Phys.Rev.Lett. 106 (2011) 201102, [arXiv:1101.1295].

[147] R. Caldwell, A. Cooray, and A. Melchiorri, Constraints on a New Post-General Relativity Cosmological Parameter, Phys.Rev. D76 (2007) 023507, [astro-ph/0703375].

[148] E. Bertschinger and P. Zukin, Distinguishing Modified Gravity from Dark Energy, Phys.Rev. D78 (2008) 024015, [arXiv:0801.2431].

[149] Y.-S. Song and K. Koyama, Consistency test of general relativity from large scale structure of the Universe, JCAP 0901 (2009) 048, [arXiv:0802.3897].

[150] Y.-S. Song, L. Hollenstein, G. Caldera-Cabral, and K. Koyama, Theoretical Priors On Modified Growth Parametrisations, JCAP 1004 (2010) 018, [arXiv:1001.0969].

[151] Y.-S. Song, G.-B. Zhao, D. Bacon, K. Koyama, R. C. Nichol, et. al., Complementarity of Weak Lensing and Peculiar Velocity Measurements in Testing General Relativity, Phys.Rev. D84 (2011) 083523, [arXiv:1011.2106]. 
[152] A. Hojjati, L. Pogosian, and G.-B. Zhao, Testing gravity with CAMB and CosmoMC, JCAP 1108 (2011) 005, [arXiv:1106.4543].

[153] T. Baker, P. G. Ferreira, C. Skordis, and J. Zuntz, Towards a fully consistent parameterization of modified gravity, Phys.Rev. D84 (2011) 124018, [arXiv:1107.0491].

[154] G.-B. Zhao, H. Li, E. V. Linder, K. Koyama, D. J. Bacon, et. al., Testing Einstein Gravity with Cosmic Growth and Expansion, arXiv:1109.1846.

[155] J. Zuntz, T. Baker, P. Ferreira, and C. Skordis, Ambiguous Tests of General Relativity on Cosmological Scales, arXiv:1110.3830.

[156] Euclid Consortium Collaboration, L. Amendola, S. Appleby, D. Bacon, T. Baker, et. al., Cosmology and fundamental physics with the Euclid satellite: Review Document of the Euclid Theory Working Group, . in preparation.

[157] M. Kunz, The dark degeneracy: On the number and nature of dark components, Phys.Rev. D80 (2009) 123001, [astro-ph/0702615].

[158] W. Hu and D. J. Eisenstein, The Structure of structure formation theories, Phys.Rev. D59 (1999) 083509, [astro-ph/9809368].

[159] M. Kunz, Why we need to see the dark matter to understand the dark energy, J.Phys.Conf.Ser. 110 (2008) 062014, [arXiv:0710.5712].

[160] The SNLS Collaboration Collaboration, P. Astier et. al., The Supernova legacy survey: Measurement of omega $(m)$, omega (lambda) and $W$ from the first year data set, Astron.Astrophys. 447 (2006) 31-48, [astro-ph/0510447].

[161] WMAP Collaboration Collaboration, D. Spergel et. al., Wilkinson Microwave Anisotropy Probe (WMAP) three year results: implications for cosmology, Astrophys.J.Suppl. 170 (2007) 377, [astro-ph/0603449].

[162] L. Amendola and S. Tsujikawa, Phantom crossing, equation-of-state singularities, and local gravity constraints in $f(R)$ models, Phys.Lett. B660 (2008) 125-132, [arXiv:0705.0396].

[163] G. Huey and B. D. Wandelt, Interacting quintessence. The Coincidence problem and cosmic acceleration, Phys.Rev. D74 (2006) 023519, [astro-ph/0407196].

[164] S. Das, P. S. Corasaniti, and J. Khoury, Super-acceleration as signature of dark sector interaction, Phys.Rev. D73 (2006) 083509, [astro-ph/0510628].

[165] R. Hlozek, M. Cortes, C. Clarkson, and B. Bassett, Non-parametric Dark Energy Degeneracies, arXiv:0801.3847.

[166] C. Clarkson, M. Cortes, and B. A. Bassett, Dynamical Dark Energy or Simply Cosmic Curvature?, JCAP 0708 (2007) 011, [astro-ph/0702670].

[167] C. Clarkson, B. Bassett, and T. H.-C. Lu, A general test of the Copernican Principle, Phys.Rev.Lett. 101 (2008) 011301, [arXiv:0712.3457].

[168] R. Maartens, T. Gebbie, and G. F. Ellis, Covariant cosmic microwave background anisotropies. 2. Nonlinear dynamics, Phys.Rev. D59 (1999) 083506, [astro-ph/9808163].

[169] A. Vainshtein, To the problem of nonvanishing gravitation mass, Phys.Lett. B39 (1972) 393-394.

[170] J. Khoury and A. Weltman, Chameleon fields: Awaiting surprises for tests of gravity in space, Phys.Rev.Lett. 93 (2004) 171104, [astro-ph/0309300].

[171] P. Brax, C. van de Bruck, A.-C. Davis, J. Khoury, and A. Weltman, Detecting dark energy in orbit - The Cosmological chameleon, Phys.Rev. D70 (2004) 123518, [astro-ph/0408415].

[172] W. Hu and I. Sawicki, Models of $f(R)$ Cosmic Acceleration that Evade Solar-System Tests, Phys.Rev. D76 (2007) 064004, [arXiv:0705.1158]. 
[173] G.-B. Zhao, B. Li, and K. Koyama, Testing General Relativity using the Environmental Dependence of Dark Matter Halos, Phys.Rev.Lett. 107 (2011) 071303, [arXiv:1105.0922].

[174] J.-P. Uzan, C. Clarkson, and G. F. Ellis, Time drift of cosmological redshifts as a test of the Copernican principle, Phys.Rev.Lett. 100 (2008) 191303, [arXiv:0801.0068].

[175] J. Yoo, General Relativistic Description of the Observed Galaxy Power Spectrum: Do We Understand What We Measure?, Phys.Rev. D82 (2010) 083508, [arXiv:1009.3021].

[176] C. Bonvin and R. Durrer, What galaxy surveys really measure, Phys.Rev. D84 (2011) 063505, [arXiv:1105.5280].

[177] A. Challinor and A. Lewis, The linear power spectrum of observed source number counts, Phys.Rev. D84 (2011) 043516, [arXiv:1105.5292].

[178] D. Jeong, F. Schmidt, and C. M. Hirata, Large-scale clustering of galaxies in general relativity, arXiv:1107.5427. 